\title{
THE AMOUNTS AND ADEQUACY OF FOOD RATIONS IN FINLAND DURING 1941-46
}

\author{
PaAvo Roine \\ Biochemical Institute, Helsinki
}

Received 3. III. 1948.

The food rationing in Finland was introduced progressively beginning in the autumn of 1939 when sugar and coffee were put on cards. Bread was rationed in the spring of 1940 , milk, meat, and fats, including butter, margarine, lard, and cheese, in the autumn of 1940. The rationing of eggs and fish was started early in 1941. The only foodstuff which in addition to those mentioned above has greater importance in the Finnish dietary is potatoes, which were rationed only during shorter periods.

In the division of the population into ration groups a great difference has prevailed between the "self-providers," i.e., farmers and their families, and the ordinary "consumers." The rations of the self-providers have been remarkably higher in many foodstuffs than those of the consumers and, in addition, the control of the food consumption in the farmers' families is, of course, very difficult. It can be assumed that their caloric food intake has been about the same as in normal times, and because milk, as a cheap food, has evidently been consumed in ample amounts, there is no reason to assume that any greater deficiencies have occurred in the selfproviders' nutrition. The consumers, on the other hand, were forced to live chiefly upon their ordinary card rations, the amount of the products obtained from the "black market» evidently being, at least during the first years of the rationing, relatively little. The real food consumption of this great group of people, about two thirds of the Finnish population, can therefore be calculated with rather good accuracy, and a picture of the nutrition situation during the years of rationing can thus be obtained. It should be mentioned here that Finnish diets during the first years of rationing have earlier been dealt with by VIRTANEN $(19,20)$ and by Simola (16).

In this paper are given the daily rations of the most important foodstuffs distributed to the consumers during the years 1941-46. On the basis of these the caloric values as well as the protein, fat, calcium, phosphorus, iron, vitamin A, thiamine, riboflavin, niacin, and ascorbic acid contents have been calculated. 
Table I. The Amounts of Different Constituents in Foodstuffs

Values of fresh raw foodstuffs per 100 grams of edible portion

\begin{tabular}{|c|c|c|c|c|c|c|c|c|c|c|c|}
\hline Foodstuff & $\begin{array}{c}\text { Fuel } \\
\text { value } \\
\text { Calo- } \\
\text { ries }\end{array}$ & $\begin{array}{c}\text { Pro- } \\
\text { tein } \\
\mathrm{g}\end{array}$ & $\begin{array}{c}\text { Fat } \\
\mathrm{g}\end{array}$ & $\begin{array}{c}\text { Cal- } \\
\text { cium } \\
\text { mg }\end{array}$ & $\begin{array}{c}\text { Phos- } \\
\text { phorus } \\
\mathrm{mg}\end{array}$ & $\begin{array}{l}\text { Iron } \\
\text { mg }\end{array}$ & $\begin{array}{c}\text { Vita- } \\
\text { min A } \\
\text { value } \\
\text { I. U. }\end{array}$ & $\begin{array}{l}\text { Thia- } \\
\text { mine } \\
\text { mg }\end{array}$ & $\begin{array}{c}\text { Ribo- } \\
\text { flavin } \\
\text { mg }\end{array}$ & $\begin{array}{c}\text { Niacin } \\
\text { mg }\end{array}$ & $\begin{array}{c}\text { Ascor- } \\
\text { bic } \\
\text { acid } \\
\text { mg }\end{array}$ \\
\hline Rye Flour $\ldots \ldots$ & 354 & 10.0 & 1.1 & 33 & 289 & 4.6 & 50 & 0.29 & 0.14 & 0.6 & - \\
\hline $\begin{array}{r}\text { Wheat Flour }(0.9 \% \\
\text { ash content) }\end{array}$ & 351 & 12.1 & 2.0 & 44 & 215 & 2.1 & 30 & 0.24 & 0.10 & 2.0 & - \\
\hline Milk (in summer).. & 70 & 3.0 & 4.0 & 120 & 93 & 0.05 & 200 & 0.04 & 0.18 & 0.1 & 1.5 \\
\hline " (in winter) $\ldots$ & 70 & 3.0 & 4.0 & 120 & 93 & 0.05 & 70 & 0.04 & 0.18 & 0.1 & 1.5 \\
\hline Butter (in summer) & 785 & 0.7 & 83.7 & 15 & 17 & 0.2 & 4200 & $\cdot$ & - & - & - \\
\hline$" \quad$ (in winter) & 785 & 0.7 & 83.7 & 15 & 17 & 0.2 & 1500 & - & - & - & - \\
\hline Margarine...... & 791 & 0.5 & 84.6 & 3 & 1 & 0.2 & - & - & - & 一 & - \\
\hline Sugar $\ldots . . . \ldots \ldots$ & 410 & - & - & - & - & - & - & - & - & - & - \\
\hline Beef, lean $\ldots . . .$. & 156 & 20.0 & 8.0 & 12 & 216 & 2.5 & 50 & 0.13 & 0.20 & 3.8 & - \\
\hline Potatoes (in autumn) & 96 & 2.0 & 0.2 & 10 & 41 & 0.75 & 50 & 0.07 & 0.05 & 0.5 & 17 \\
\hline " (in spring) & 96 & 2.0 & 0.2 & 10 & 41 & 0.75 & 50 & 0.07 & 0.05 & 0.5 & 10 \\
\hline
\end{tabular}

There are, of course, many other food constituents which are of importance for human nutrition, but it can be assumed that when the better known factors are present in adequate quantities, these constituents also are provided in sufficiency.

In Table 1 there are given the values which have been used in this investigation in calculating the amounts of different constituents in several foodstuffs. All the values have been taken from literature, using, if possible, the results of analyses made in Finland from native foodstuffs. The main part of the values is from the extensive investigation of VIRTANEN and TURPEINEN (23) on the nutrition of people relatively poor in Finland during 1936 - 37. The iron values are from TurPEINEN (18), the thiamine and niacin contents of cereals from PULKKI $(11,12,13)$, and the thiamine content of potatoes, the niacin content of meat and potatoes, and the ascorbic acid content of potatoes from Simola (17). Additional facts have been taken from Sherman (15), Peterson, Skinner and Strong (10), Eddy and Dalldorf (3), Lunde (8), Virtanen and Holmberg (21), Kent-Jones and Amos (5), and Bacharach and Rendle (1).

The figures given in Table 1 are the total values of the constituents; the extent to which they are absorbed and utilized by the body has not been taken into consideration. The values are, of course, only approximate because the quality of the foodstuffs can vary considerably. In this respect the following remarks are to be made:

Rye Flour. The whole rye meal commonly used in Finland was during the years, investigated mixed with small amounts of barley, wheat bran, etc., which cause some variations in the composition of the rye flour. In addition to rye, small amounts of peas, oats, macaroni, etc., have been distributed on the »black bread coupons.» The errors made in calculating the values only on the basis of rye flour can, however, not be significant. 
The wheat flour used during 1941-46 was long-extraction flour, the ash content of which averaged to $0.9 \%$.

The vitamin A values of milk and butter are known to fluctuate greatly at different seasons and therefore different values have been used for these products in summer and in winter.

The meat ration was dependent on price, so that more was obtained of cheaper qualities than of expensive. The values in the tables are, however, calculated only for beef, which was the most used of the different kinds of meat.

In potatoes considerable losses of ascorbic acid occur during the preservation, therefore different values have been used in spring and in autumn.

\section{Daily Amounts and Adequacy of Different Food Constituents}

In Tables $2-7$ are given the average daily rations of the most important foodstuffs during $1941-46$ as well as the calculated values of different constituents. The division of the people into different groups has been made according to the grouping used for the distribution of the ration cards. During the years this grouping has been somewhat changed.

In addition to the ordinary rationed food, potatoes, too, have been taken into consideration, due to their great importance in the dietary. The amounts of potatoes consumed are, of course, very difficult to evaluate, especially because the supply was at times very scarce. During the periods when potatoes were rationed, the daily rations varied from 350 to 600 grams. Because, however, the consumers had the possibility of reserving potatoes in advance, it is probable that the consumption was remarkably greater also in these times. On the whole it can be assumed that people have been able to consume potatoes in the desired amounts. In Tables $2-7$ some average values have been presumed to have been consumed and their energy, etc., contents have been added to the values obtained from the rationed foodstuffs.

Calories. The caloric value of the rationed foodstuffs of an adult normal consumer during $1941-46$ varied from 1130 to 1440 Calories per day. Only about one half of the energy requirement could thus be covered by ordinary rations. The deficit was filled chiefly by potatoes, and to a smaller extent by fish, rutabegas, carrots, etc. The foodstuffs obtained from the »black market» have also, of course, played some role.

The energy situation of children has been quite satisfactory up to 9 years of age; up to 3 years adequate amounts of calories were obtained in the rationed foodstuffs alone and from 4 to 9 years a moderate intake of potatoes was able to make the caloric values sufficient. From 10 years upwards, however, the share of the rationed foodstuffs in supplying the required calories has been much smaller, and it has been difficult to reach the daily requirements unless rather large amounts of potatoes and other available foodstuffs have been eaten. 
In the group of light workers the energy contents of the daily rations varied from 1330 to 1680 Calories, with heavy workers from 1730 to 1960 Calories, and with very heavy workers from 1950 to 2200 Calories. To reach the nutritional standards in these groups at least the second half of the necessary calories had to be obtained from ration-free foodstuffs. As can be seen from the Tables, daily intakes of, for example 1200 grams of potatoes among heavy, and 1500 grams among very heavy workers have raised the energy values only to $2900-3100$ and $3400-3900$ Calories respectively. To reach 4000 and 5000 Calories respectively remarkable amounts of additional foodstuffs have thus been necessary. If they were not obtained, the so-called very heavy work must have changed to heavy work, heavy to light work, and so forth.

Protein. The amount of protein in the normal consumer's daily rations has varied from 31.2 to 44.2 grams, from which amounts 30 to $40 \%$ consisted of animal protein. These values are much lower than are, for example, the recommended daily allowances of the National Research Council, U.S.A. (70 grams for men of $70 \mathrm{~kg}$, 60 grams for women of $56 \mathrm{~kg}$ ). Considering the amounts obtained in potatoes and eventually from other additional foodstuffs, it must, however, be concluded that the amount of protein actually required by an adult person has been reached. The percentage of animal protein is relatively small, but attention must be paid to the fact that the major part of the plant protein taken has been whole meal protein, the biological value of which evidently is better than that of white flour (cf. СНICK, 2 ). In the groups of workers with larger daily rations, the protein situation has been more advantageous than that of the normal consumers, because the protein requirement is independent of the kind of work.

Children up to 3 years of age have obtained the recommended standard allowances of protein in the rationed foodstuffs alone. When a moderate consumption of potatoes ( 300 grams per day) is taken into consideration, the daily protein intake of children aged $4-6$ years has also risen to the recommended allowance (50 grams). From 7 years upwards, however, the daily amounts have remained under the recommended allowances, even though differences have not been very remarkable. The most serious situation has existed in the age group of 19-20 years, whose protein requirements are very high ( $75-100$ grams) and whose milk ration has been only the same as that of the normal consumers.

Fat. The knowledge of the requirements of fat in general are as yet very uncertain, although it is known that some unsaturated fatty acids are nutritionally essential. In normal times the fat consumption has been over 100 grams per capita per day in some countries, while people in some other countries have consumed only 30 grams or less per day. Usually it is considered that people doing heavy manual work in cold climate need large quantities of fat. As can be seen from the Tables the Finnish wartime diets have been relatively poor in fat. The normal consumers' daily rations have contained about 30 grams of fat, those of the heavy workers about 40 grams. The fat contents of the children's diets have amounted to $50-60$ grams. 
Calcium. The amount of calcium in the rations of the normal consumers has varied from 0.31 to 0.43 grams. ${ }^{1}$ It has been particularly low, $0.31-0.36$, during the years when the daily milk ration was only $200-225$ grams. Because potatoes are poor in calcium and the role of green leaf vegetables in the Finnish winter dietary is insignificant, it can be seen that the daily calcium intake must have been much below the recommended allowance of 0.8 grams and, at least during some years, also below the minimum requirements. The situation could seem more dangerous still, considering the fact that the bread flour ration consists largely of rye whole meal with high phytic acid content. Due to the manner of preparing bread, however, the most part of phytic acid is enzymically broken down (cf. MøLLGAARD, Lorenzen, Hansen and Christensen, 9) having probably no very harmful effect on the absorption of calcium. In any case it can be stated that the normal consumers' diets during 1941-46 have been clearly deficient in calcium.

Some deficiency can be noticed also in children's diets. Only children under 1 year of age (during 1944-46, under 2) whose milk ration was $900-1000 \mathrm{ml}$ have obtained the recommended allowance of calcium. From 1 (or 2) years upwards the daily milk ration was $600 \mathrm{ml}$ and the total amount of calcium obtained in the rationed foodstuffs averaged $0.8-0.9$ grams. The intake of calcium has been inadequate probably at least for adolescents girls and boys, whose recommended daily allowance is 1.3 to 1.4 grams. Grossly deficient in calcium has evidently been the diet for children of 19-20 years of age, who obtained only the normal consumers' rations.

The calcium intakes of the heavy and very heavy workers have been somewhat higher than those of the normal consumers, but also they have remained at a relatively low level.

The deficiency of calcium led in the antumn of 1945 to giving the order that about $0.5 \%$ of calcium as calcium carbonate was to be added to the rye bread produced by bakeries.

Phosphorus. The values of the phosphorus requirements given in literature are somewhat variable, but evidently about one gram should supply the daily need of an adult person for normal maintenance. Children need for optimal growth and development somewhat more, probably about 1.3 grams. It can be seen from the Tables that adults' diets have contained adequate amounts of phosphorus and the same holds true also for children under 1 year and over 4 years of age. For the $1-3$ years' group the allowance of phosphorus has been somewhat scanty.

Iron. The recommended allowances of the National Research Council (U.S.A.) for iron are $12 \mathrm{mg}$ for adults, and $6-15 \mathrm{mg}$ for children. TuRPEINEN (18), however, reports much lower values, viz., $1 \mathrm{mg}$ for men, $5 \mathrm{mg}$ for women and $2-8 \mathrm{mg}$ for children. The amount of iron obtained from rationed foodstuffs alone has been 9.6 - $12.4 \mathrm{mg}$ for normal consumers, and $6.1-14.9 \mathrm{mg}$ for children of different

i) In addition drinking water contains small, largely varying amounts of calcium. If the average calcium content of Finnish waters is taken for $0.02 \mathrm{~g} / 1$ and the normal daily consumption for 3 liters, the calcium obtained in water amounts to 0.06 grams. 
ages. According to these values the iron intake can be regarded as wholly adequate or even ample.

Vitamin A. The vitamin A content of the diets has been very low throughout. The allowances of the normal consumers have varied in summer from 1050 to 1690 and in winter from 440 to 680 International Units. All these values have risen to some extent when potatoes also are taken into consideration. In summer and in autumn green and yellow vegetables may have increased the vitamin A potency of the diets, and also in winter carrots may have been eaten to such an extent that they could have some significance in the vitamin A maintenance, if only theit carotene were well absorbed. VAN EEkELEN and PAnnevis (4) showed, however, that of the carotene of carrots fed to human beings $99 \%$, and of that of spinach $94 \%$, were excreted in the faeces. Also Kreula and Virtanen (6) as well as VirtaNEN and KREULA (22) have shown that on the average only $10 \%$ of the carotene of carrots is absorbed by the body. The vitamin A potency of the vegetable foods can thus not be judged simply on the basis of their carotene contents and the values found in literature are evidently in many cases too high. In calculating the vitamin A potency of a diet on the basis of its carotene content, the material from which the carotene is obtained ought always to be known.

The optimal vitamin A supply of an adult person is held to be about 3500 5000 International Units. As can be seen from the Tables all Finnish adults' diets during 1941-46 have contained only a small part of this requirement. Although the bulk of the amount obtained has been in readily absorbable form (in milk and in butter), it must be stated that the diets, especially during the winter, have been grossly deficient in vitamin A.

Due to greater milk rations and lower requirements, the situation of children has not been so bad as that of the adults. Children of $1-9$ years of age - as well as the children under 1 year of age, whose vitamin $\mathrm{A}$ allowance has always been quite satisfactory - have during summer seasons probably obtained vitamin A in adequate quantities. During winter the diets of these children have been deficient in vitamin A, and this holds true for older children, particularly, whose optimal supplies are held to amount to 4000 - 6000 International Units.

Some improvement in the vitamin A situation during winter came about through feeding a considerable part of Finnish cattle with A.I.V. silage, because the milk thus produced has a high vitamin A potency throughout the year.

Thiamine. The thiamine contents of the daily rations of the normal consumers during 1941-46, amounts according to the Tables to $0.7-0.9 \mathrm{mg}$, the actual allowances, however, being somewhat lower because about $15 \%$ of the flour-thiamine is destroyed in the manufacture of bread (PulkKI and PuUtula, 14). If in addition to the rations 500 grams of potatoes are consumed, the daily amount of thiamine rises to $1.1-1.3 \mathrm{mg}$, i.e., to values close to the recommended daily allowances. Although it is not satisfactorily known to what extent the thiamine of certain foodstuffs is absorbed by the human body, it seems evident that at least the minimum requirements of thiamine (for a normal consumer about $1 \mathrm{mg}$ ) are met with 
in the amounts obtained. The same holds true also for children and workers whose thiamine intake during the time investigated must be regarded as having been adequate. The increased need of more active consumers has been filled by the thiamine of larger card rations and by the increased consumption of potatoes.

Riboflavin. The great importance of riboflavin to health, efficiency, vigor, and resistance, has been clearly demonstrated only in recent years, when also the daily requirements for this vitamin have been estimated. If the amounts of riboflavin obtained in Finnish diets during 1941-46 are compared with the recommended allowances of National Research Council (U.S.A.), it can be seen that the riboflavin supply of adults' diets has been very scanty throughout, resulting chiefly from the small milk rations during these years. Thus the daily amount of riboflavin in the normal consumers' rations is only $0.7-0.9 \mathrm{mg}$, and with an ample consumption of potatoes, about $1.2 \mathrm{mg}$, the recommended allowances being about $1.8 \mathrm{mg}$. The situation is as bad also among the workers with greater food consumption. Because winter diets have probably not contained eggs, green leaves, and other riboflavin-rich foodstuffs in appreciable quantities, it must be assumed that riboflavin deficiency has actually existed in Finland during the years investigated.

The children's situation has been better due to larger milk rations. The supply of riboflavin has been quite adequate up to the age of 9 years and sufficient even for the older children. The most severe deficiency has occurred with boys of 19-20 years, whose recommended riboflavin allowance is $2.5 \mathrm{mg}$ but whose diets hardly contained one half of that amount.

Niacin (Nicotinic Acid). The present knowledge of the niacin requirements in human nutrition is very uncertain. Recommended allowances have, however, been given, varying from 11 to $20 \mathrm{mg}$ daily for adults, and from 4 to $18 \mathrm{mg}$ for children. It can be seen from the Tables that the amounts of niacin obtained in the Finnish diets during 1941-46 have been very small. It is possible, however, that the value of the niacin content of potatoes $(0.5 \mathrm{mg}$ per $100 \mathrm{~g})$ used in the calculations, is too low and that the amounts actually obtained in the diets are greater. In any case it seems that the supply of niacin has been rather scanty.

Ascorbic Acid. Of the rationed foodstuffs only milk has contained notable amounts of ascorbic acid, and therefore the amount of this vitamin in ordinary rations has been very small. The chief source of ascorbic acid in the Finnish dietary, especially during winter and spring, are the potatoes, which generally are consumed in rather large quantities. Particularly during the wartime shortage of other foodstuffs the consumption of potatoes was extraordinarily great, and their importance as sources of ascorbic acid quite decisive. Owing to the different varieties of potatoes, losses during preservation, and losses during food preparation, reliable information about the actual amounts of ascorbic acid contained in the diets is difficult to obtain. The average values of ascorbic acid in the potatoes taken as a basis for the calculations in this paper are probably not too high, at least, (e.g. LOJANDER, 7, has reported even 
$45 \mathrm{mg}$ ascorbic acid in cooked Finnish potatoes). The calculated amounts of ascorbic acid given in the Tables show that if potatoes have been eaten in moderate quantities, the amounts of ascorbic acid have in general filled at least the minimum requirements. The values for children under 1 year of age seem to be very low, but it ought to be kept in mind that the ascorbic acid content of mothers' milk is much higher than that of cow's milk, and that certain berry juices are usually fed to babies.

Children and Adults. Examination of the food rations and of the adequacy of several constituents shows that the children have been during 1941-46 in a much better position than the adults. The nutrition of the children under 1 year of age has evidently been fully adequate, considering the calories and the protein as well as the mineral elements and the vitamins. The diets of children up to about 9 years of age, too, have been adequate as regards the calories and most of the food constituents, but some shortage may have occurred in the amounts of calcium, and a distinct deficiency can be noticed during winter in the amounts of vitamin A. From 10 years upwards the caloric values of the diets have been somewhat under the recommended standard allowances, the supply of protein has been scanty, and a distinct deficiency has occurred in the amounts of calcium and vitamin A. The extra rations of grain products, milk, margarine or butter, and sugar, which have been distributed to institutions such as kindergartens, children's homes, children's summer colonies, school kitchens, and juvenile summer camps have, however, brought about remarkable improvements in the nutrition of children. Distribution of vitamin preparations, particularly that of cod-liver oil, has also improved children's nutrition.

In the adults' diets several deficiencies can be noticed. A shortage of calories is very clear, particularly in those groups whose energy requirements are great, a fact which evidently must have decreased the capacity for performing heavy work. Calcium deficiency is also general, being worst among the normal consumers. Of the vitamin deficiencies that of vitamin A is most severe; especially have the diets been grossly deficient in this vitamin during the winter periods. A slight deficiency of riboflavin, and possibly also of niacin can still be noticed in adults' dietaries. The deficiencies of calcium and vitamin A in the Finnish diets in the end of 1941 were stressed already by VIRTANEN (20) in 1942 .

A striking fact about adults' diets during $1941-46$ is the smallness of the milk rations compared with the amounts of milk consumed in Finland in years before the war. The decrease in the milk consumption has caused the worst deficiencies in the wartime nutrition. The shortage of calcium as well as that of riboflavin could easily be avoided with larger milk rations. The vitamin A situation which has been nearly exclusively dependent on milk and butter, could also be thoroughly changed with larger milk rations, especially if the vitamin A and carotene contents of milk during winter and spring were kept on a high level with A.I.V. silage feeding.

A comparison of the food rations in different years shows that the adequacy of different constituents has somewhat changed depending on the amounts of the 
foodstuffs distributed. Detailed knowledge on the amounts of calories, vitamins, etc., obtained during different years and in different groups of consumers can be seen from the figures given in the Tables. If, however, in order to get a general picture of the development of nutrition, the diets of the normal consumers in the years 1941-46 are compared, it can be stated that the situation was worst during 1942. The amounts of calories as well as of all the other food constituents were during this year at their lowest level, the people were generally starving, and the food was grossly deficient in different constituents. The next worst wave trough was reached in 1945 after the end of the war, but the year 1946 was already remarkably better. In 1947 meat was freed from the rationing, and the food situation developed in some other respects, too, in a more favourable direction.

\section{Summary.}

Owing to the impossibility of obtaining reliable information on the food consumption of the "self-providers," only the food rations and nutrition of the ordinary "consumers" are discussed in this paper.

The daily amounts of calories, protein, fat, calcium, phosphorus, iron, vitamin A, thiamine, riboflavin, niacin, and ascorbic acid have been calculated on the basis of the rations of bread flour, fats, milk, sugar, and meat. Of the ration-free foodstuffs only potatoes had greater importance in the diets; their contents of different constituents have been taken into consideration in using approximate values of the daily consumption of potatoes.

Inadequacy of calories is a common feature in the diets of adults and also of adolescent children. Distinct deficiencies can also be noticed in the amounts of calcium and vitamin A, a slight deficiency in riboflavin. The intake of other constituents discussed can be regarded as being nutritionally adequate, although the amounts of protein and particularly those of niacin have been in many cases rather scanty.

The nutrition of children has been remarkably better than that of the adults, owing, above all, to the greater share of milk in the children's dietary.

The nutrition situation was the worst in 1942 and very poor again in 1945 . In 1946 and later it has been continually improving.

\section{LITERATURE}

(1) Bacharach, A. L. and Rendle, T., The Nations Food. London, 1946.

(2) CHICK, H., Biological value of the proteins contained in wheat flours. Lancet 242, 405-408, 1942. Ref. Nutrition Abstracts \& Revs. I2, 254, 1942.

(3) Eddy, W. H. and Dalldorf, G., The Avitaminosis. Baltimore, 3rd ed., 1944.

(4) Van Eekelen, M. and Pannevis, W., Absorptior of carotinoids from the human intestine. Nature 141, 203-204, 1938. 
(5) Kent-Jones, D. W. and Amos, A. J., Modern Cereal Chemistry. Liverpool, 4th ed. 1947.

(6) Kreula, M. and Virtanen, A. I. Absorption of carotene from carrots in humans. Upsala Läkarefören. Förh. 45, 355-362, 1939.

(7) Lojander, W., Keitetyn perunan C-vitamiinipitoisuudesta sekä sen vaihteluista. Duodecim 52 , $787-798,1936$.

(8) Lunde, G., Vitamine in frischen und konservierten Nahrungsmitteln. Berlin, II Aufl., 1943.

(9) Møllgafid, H., Lorenzen, K., Hansen, I. G. and Christensen, P. E., On phytic acid, its importance in metabolism and its enzymic cleavage in bread supplemented with calcium. Biochem. J. 40, 589-603, 1946.

(10) Peterson, W. H., Skinner, J. T. and Strong, F. M., Elements of Food Biochemistry. New York, 1945.

(11) PULKKI, L. H., Undersökningar angående spannmålens och brödets aneurinhalt i Finland. Lecture at "Nordisk cerealkemistförenings kongres i Stockholm», 1946.

(12) —- Vitamiinien määristä ja sijainnista jyvissä. Suomen Kemistilehti A 20, 197-206, 1947.

(13) $\longrightarrow$ - Personal communication, 1948.

(14) - $\rightarrow-$ and Puutula, K., Applicability of thiochrome method to determination of the thiamine content of thin hard bread and other rye products. Suomen Kemistilehti B 19, $89-90,1946$.

(15) Sherman, H. C., Chemistry of Food and Nutrition. New York, 7th ed., 1946.

(16) Simola, P. E., Nykyinen ruokamme. Kotitalousopetus 6, 31-40, 1942.

(17) — - Suomalaisten ruoba-aineiden vitamiinipitoisuudesta. Suomen Kemistilehti 18 A, 4-10, 1945.

(18) Turpeinen, O., Tutkimus suomalaisen kansanravinnon sisältämän raudan määrästä ja riittävyydestä. Diss., Helsinki, 1-116, 1946.

(19) Virtanen, A. I., Oikean ravitsemuksen järjestäminen nykyoloissa. Kotitalousopetus 4, 121124,1940 .

(20) —— Kivennäisaineiden määristä ja puutteista nykyisessä ravinnossamme. Emäntälehti $40,18-23,1942$.

(21) —- and Holmberg, T., Variations of vitamin- $B_{2}$ in milk. Suomen Kemistilehti B 11, 1, 1938

(22) —- and Kreula, M., Die Resorbierung des Carotins aus Mohrrüben beim Menschen. Z. physiol. Chem. 270, 141-152, 1941.

(23) —- - and Turpeinen, O., Tutkimus suhteellisen vähävaraisen väestön ravinnosta Suomessa 1936-37. Kansanravitsemuskomitean mietintö, 64-126, 1940.

\section{SELOST US}

\section{RUOKATAVAROIDEN SÄÄNNÖSTELYANNOKSET JA NIIDEN RIITTÄVYYS SUOMESSA VUOSINA $1941-46$}

PaAvo Roine.

Biokemiallinen Tutkimuslaitos. Helsinki.

Tutkimuksessa käsitellään kuluttajaväestön ravitsemusta vuosina 1941-46. Omavaraisen väestönosan ruokatavaroiden kulutuksesta on mahdotonta saada edes osapuilleen tarkkoja tietoja, mutta on luultavaa, että heidän ravitsemuksensa on pysynyt suunnilleen rauhanaikaisella tasolla.

Säännöstelyannosten pohjalla on laskettu eri kuluttajaryhmissä saadut päivittäiset määrät kalorioita, valkuaisaineita, rasvaa, kalsiumia, fosforia, rautaa, A-vitamiinia, tiamiinia, riboflaviinia, 
niasiinia (nikotiinihappoa) ja askorbiinihappoa. Lisäksi on otettu huomioon perunoiden sisältämät ravintoainemäärät käyttäen arviolukuja perunoiden keskimääräisestä kulutuksesta.

Taulukoissa esitettyjen lukujen pohjalla käsitellään erikseen kunkin ravintotekijän päivittäin saatuja määriä ja niiden riittävyyttä.

Aikuisten ruokavalion kaloria-arvo on ollut kauttaaltaan liian alhainen. Säännöstellyistä ruokaaineista on saatu korkeintaan puolet tarvittavasta energiamäärästä, ja energiavajaus on ollut täytettävä lähinnä perunoilla sekä osittain myös säännöstelyn ulkopuolitse saaduilla elintarvikkeilla. Ellei näitä ole kylliksi saatu, on seurauksena ollut laihtuminen tai työkyvyn aleneminen. Myöskin yli 10-vuotisten lasten ravinnossa on ilmeisesti esiintynyt energian puutetta.

Valkuaisaineiden kokonaismäärä, samoin kuin eläinvalkuaisaineiden osuus, on ollut aikuisten ravinnossa varsin niukka. Nuoremmilla lapsilla on valkuaisaineita ollut ravinnossa riittävästi, vanhemmilla sitävastoin niukasti.

Rasvan määrä aikuisten ravinnossa on ollut alhainen. Tiedot rasvan tarpeesta ovat kuitenkin toistaiseksi hyvin epävarmoja.

Kalsiumista on aikuisten ravinnossa ollut selvää puutetta, samoin kasvavien lasten ravinnossa.

Fosforin määrä on ilmeisesti ollut riittävä, raudan määrä osittain jopa runsaskin.

A-vitamiinista on ollut suurta puutetta kaikilla muilla kuluttajaryhmillä paitsi nuorimmilla lapsilla. Erityisen pahana puute on tuntunut talvisaikaan, jolloin maidon vitamiinipitoisuus on alhainen ja karotiinirikkaita kasviksia käytetään vähän.

Tiamiinin määrä ravinnossa on ilmeisesti ollut riittävä.

Riboflaviinista on ilmeisesti ollut yleistä, lievää puutetta aikuisten ravinnossa. Myöskin kasvavien lasten ravinnossa on riboflaviinia ollut niukasti.

Niasiinin määrä ravinnossa on ollut varsin alhainen.

Askorbiinihappoa on ilmeisesti saatu perunoiden ansiosta suunnilleen riittävästi.

Lasten ravinto on yleisesti ollut runsaampaa ja täydellisempää kuin aikuisten, johtuen suureksi osaksi siitä, etłä lasten maitoannokset ovat olleet huomattavasti suuremmat kuin aikuisten. Etenkin 19-20-vuotisten ravinnossa on kuitenkin ollut monia selviä puutteita.

Vuosi 1942 oli ravitsemuksen suhteen huonoin. Vuosi 1945 oli myöskin hyvin huono, mutta vuonna 1946 tilanne oli jo monessa suhteessa parantunut. 


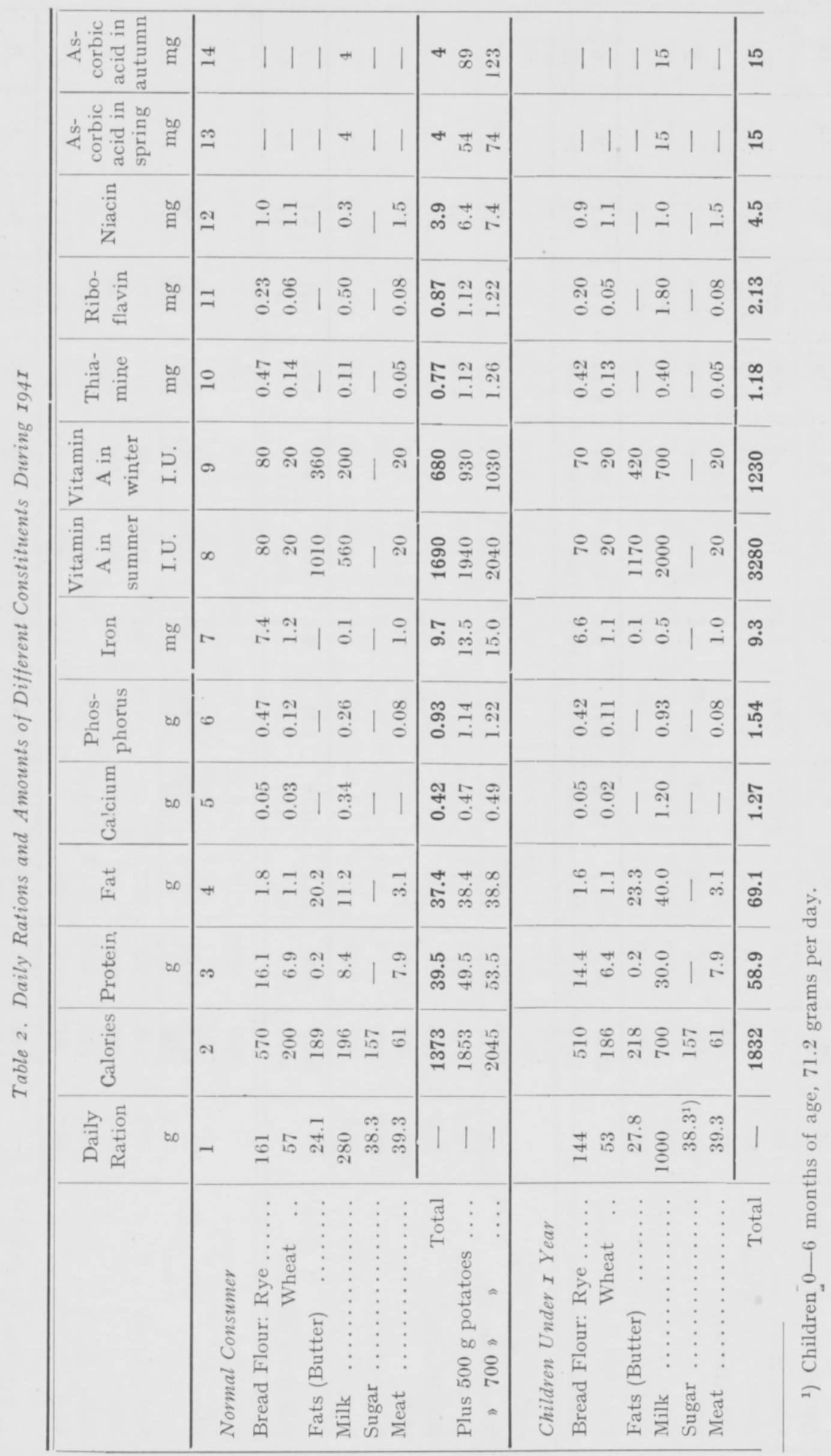




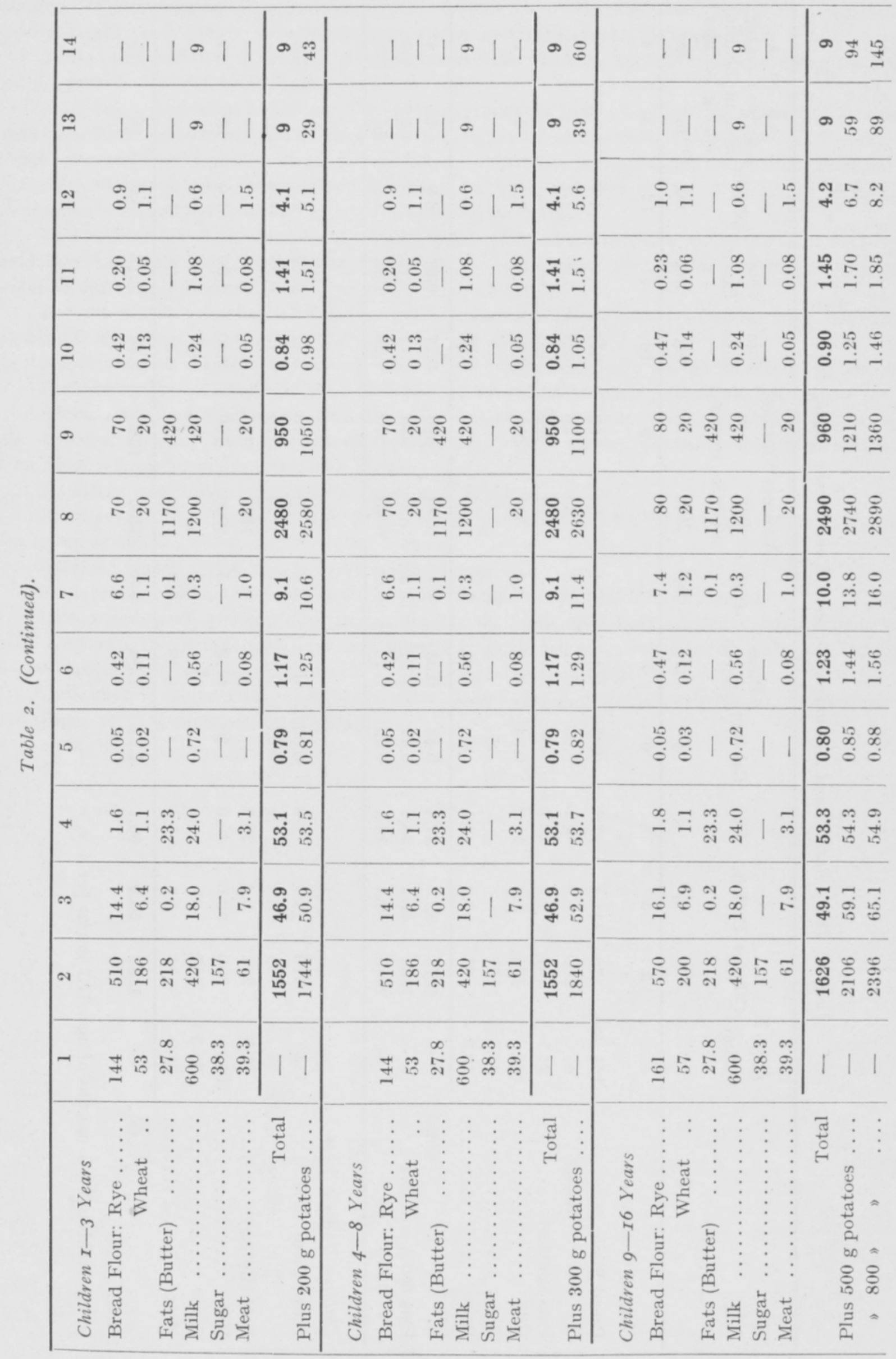




\begin{tabular}{|c|c|c|c|c|c|c|c|}
\hline & $|1| *|1|$ & 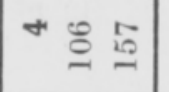 & & $1|1+1|$ & $+\underset{\sim}{+\infty}$ & $11+$ & +吾盟 \\
\hline & $\mid \begin{array}{lllll}\mid & \mid & 1 & 1\end{array}$ & + से & & $|1|+1 \mid$ & 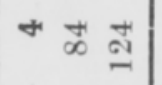 & $|1|+1$ & + ず \\
\hline & $\stackrel{+}{-}|\stackrel{\oplus}{0}| \stackrel{10}{-}$ & 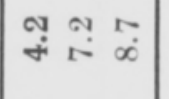 & & $\stackrel{10}{-}|\stackrel{\oplus}{0}| \overline{\sigma i}$ & $\begin{array}{lcc}0 & 0 & 0 \\
\text { in } & \stackrel{0}{0} & \stackrel{\square}{=}\end{array}$ & 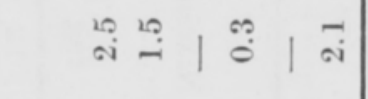 & 苟 \\
\hline & 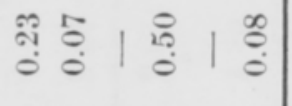 & 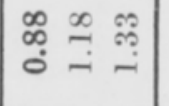 & & 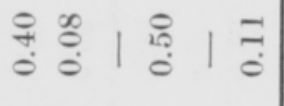 & 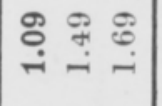 & 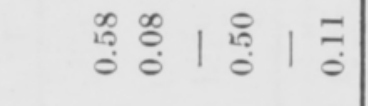 & 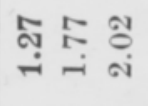 \\
\hline & 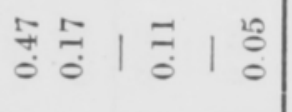 & 怘 & & 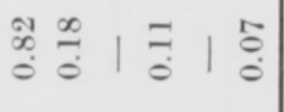 & 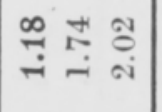 & 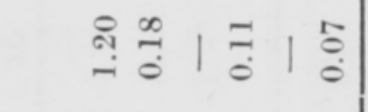 & 总 \\
\hline & 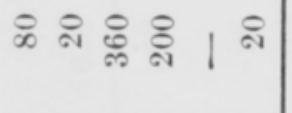 & $\begin{array}{lll}0 & 0 & 0 \\
0 & \infty & 0 \\
0 & 0 & =\end{array}$ & & 요 ఠి ఠి & 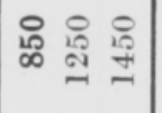 & 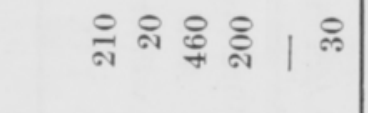 & 요욣욤 \\
\hline & 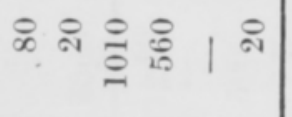 & 옹 용요 임 & & 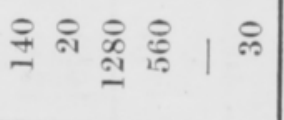 & 옹 ఝ & 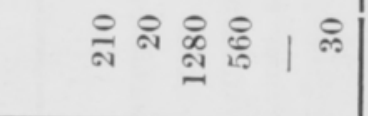 & 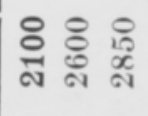 \\
\hline & 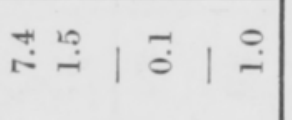 & $\begin{array}{lll}0 & 1 & \infty \\
0 & \dot{0} & 0\end{array}$ & & 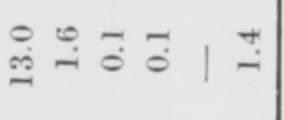 & 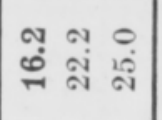 & $\vec{\sigma} \stackrel{\oplus}{-} \overrightarrow{0} \overrightarrow{0} \mid \stackrel{+}{-}$ & 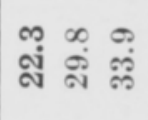 \\
\hline & 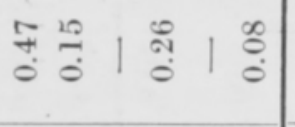 & 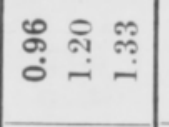 & & 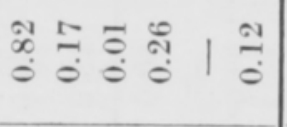 & 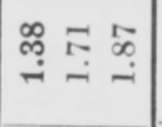 & 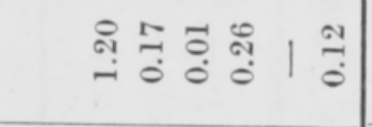 & 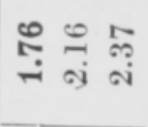 \\
\hline & 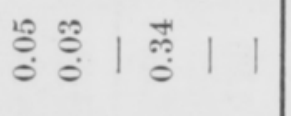 & 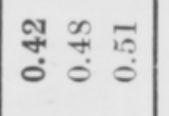 & & 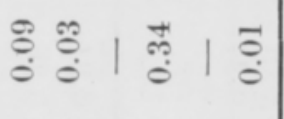 & 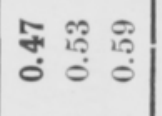 & 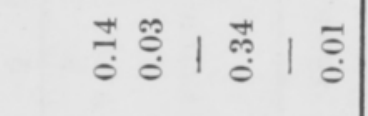 & 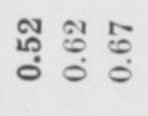 \\
\hline & 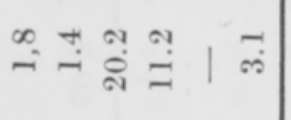 & 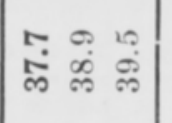 & & 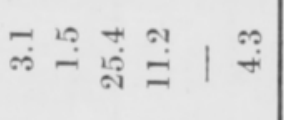 & 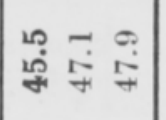 & 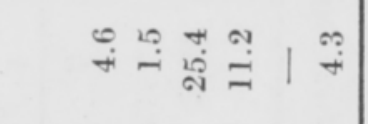 & $\begin{array}{l}0 \\
\text { 小ं }\end{array}$ \\
\hline & 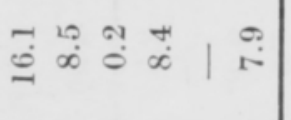 & 字 & & 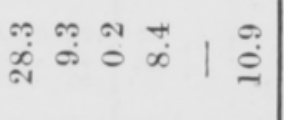 & 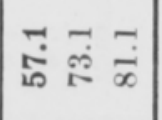 & 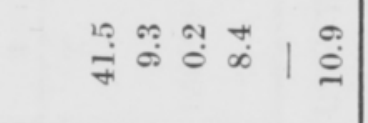 & 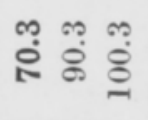 \\
\hline & 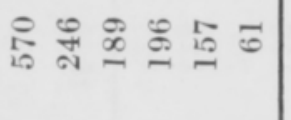 & 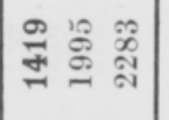 & & 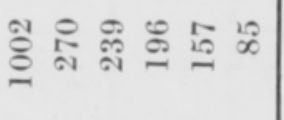 & 索学 & 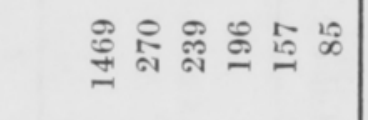 & 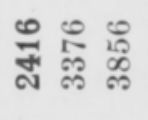 \\
\hline & ঢ一 & 111 & & 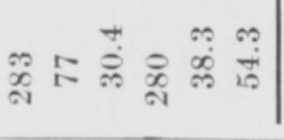 & 111 & 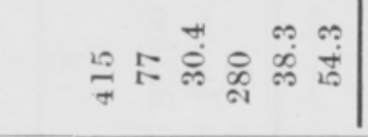 & 11 \\
\hline 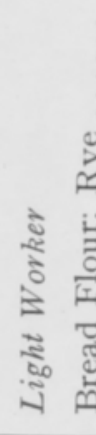 & 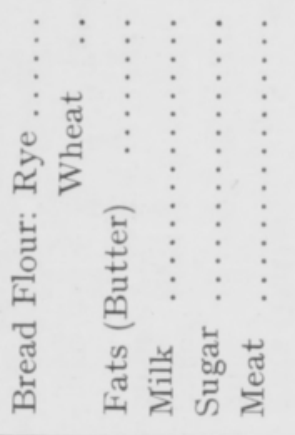 & 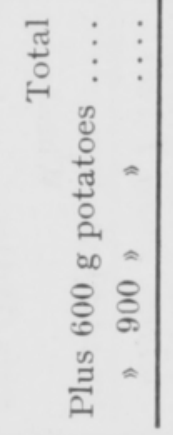 & 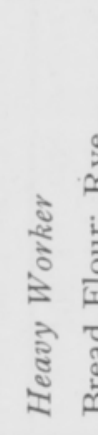 & 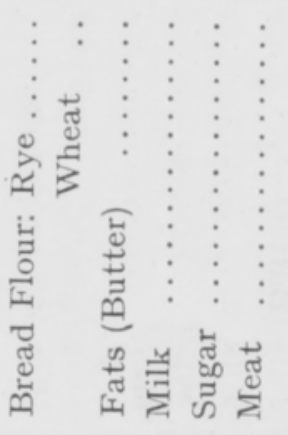 & 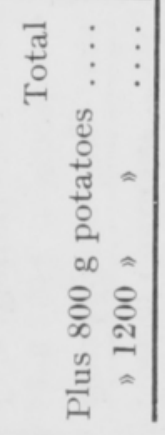 & 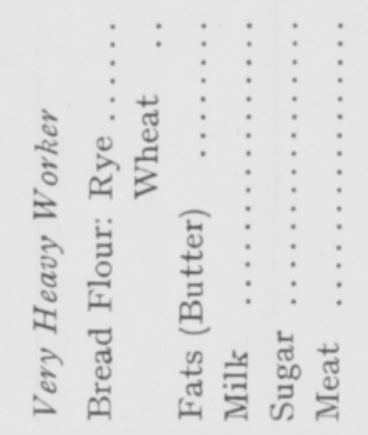 & 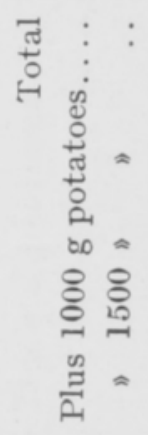 \\
\hline
\end{tabular}




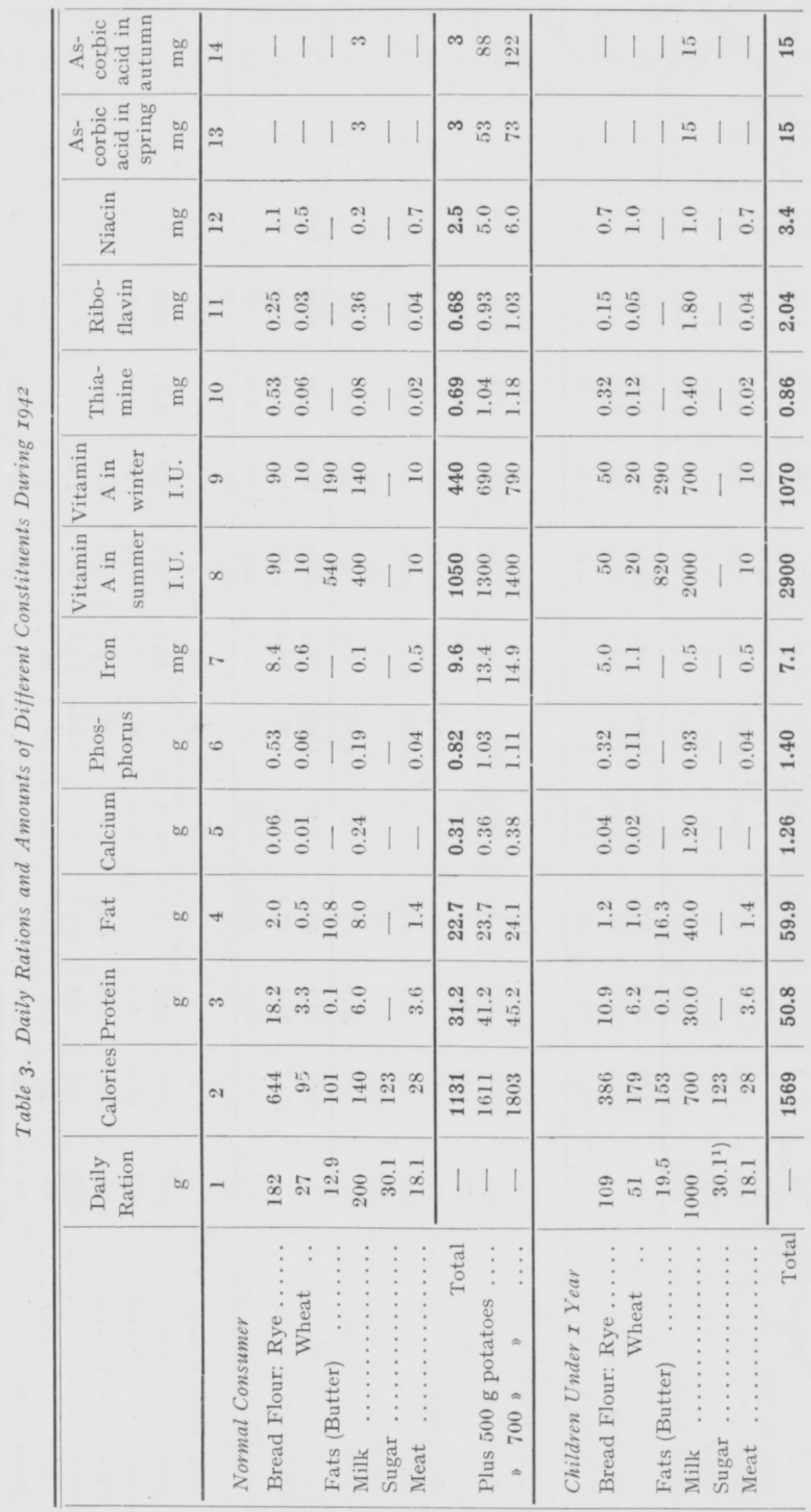




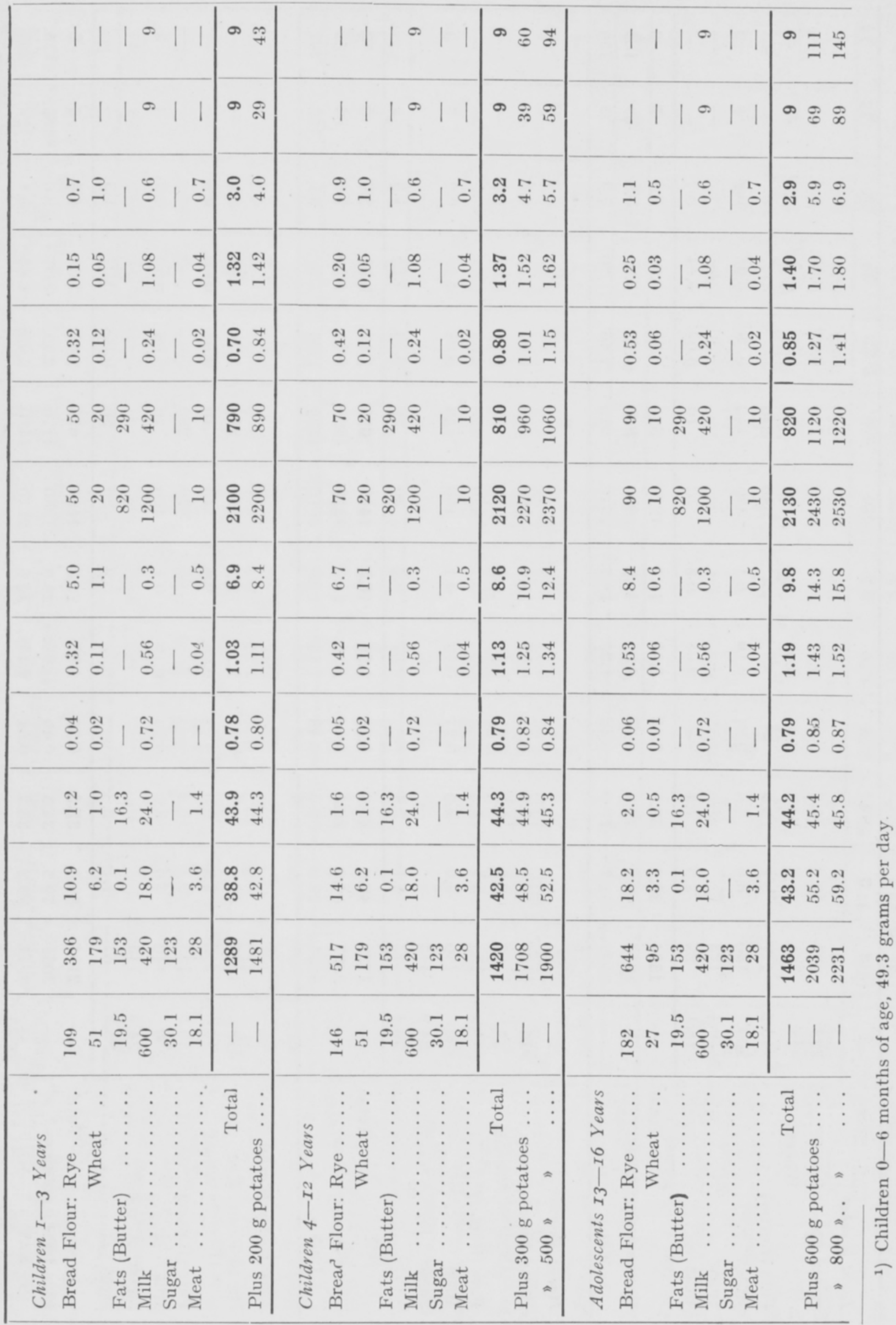




\begin{tabular}{|c|c|c|c|c|c|c|}
\hline \pm & 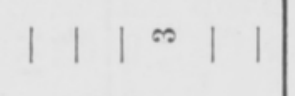 & 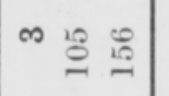 & $\left|\begin{array}{llll}\mid & \mid & \infty & \mid\end{array}\right|$ & m & ||$|\cdots| \mid$ & 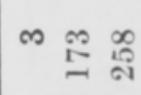 \\
\hline$\cong$ & $\left|\begin{array}{lllll}\mid & \mid & \mid & \infty & 1\end{array}\right|$ & $\infty \ddot{g}$ & ||$|\infty| 1$ & 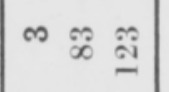 & $|1| \infty \mid 1$ & $\infty \stackrel{⿰ \zh90}{\varrho}$ \\
\hline$\stackrel{9}{9}$ & 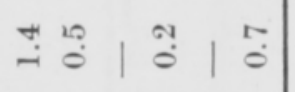 & 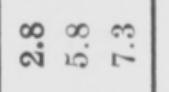 & 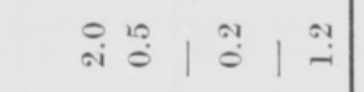 & 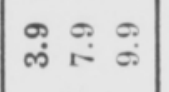 & 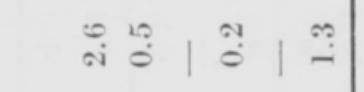 & 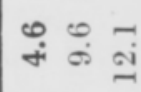 \\
\hline$=$ & 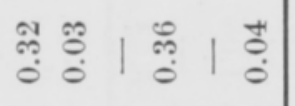 & مَ & 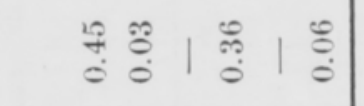 & 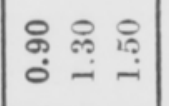 & 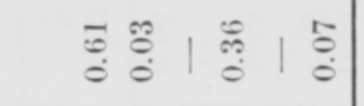 & 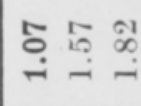 \\
\hline$\varrho$ & 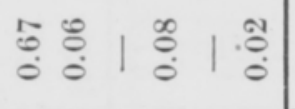 & ஜ & $\begin{array}{lllll} & 0 & 0 & 0 & 0 \\
0 & 0 & \mid & 0 & 0 \\
0 & \mid & 0 \\
0\end{array} \mid$ & 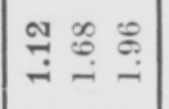 & 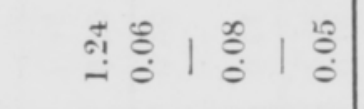 & 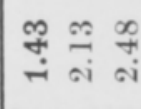 \\
\hline o & 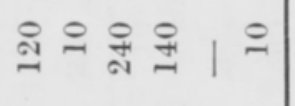 & 융 유용 & 으요요요 & 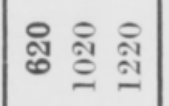 & 읏요요요요 & 웅요ㅇㅝㅛ \\
\hline$\infty$ & 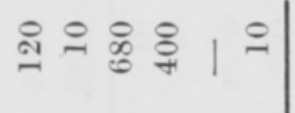 & 오요요 & હ & 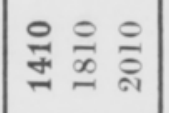 & 오용요 & 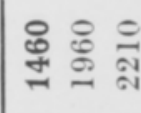 \\
\hline$r$ & 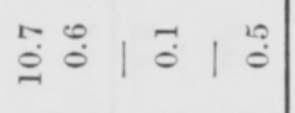 & $\begin{array}{l}\ddot{+}+r \\
\stackrel{0}{=} \stackrel{0}{=}\end{array}$ & 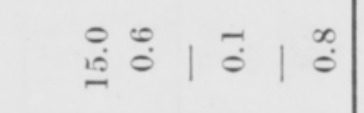 & 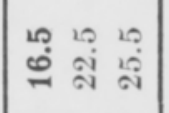 & 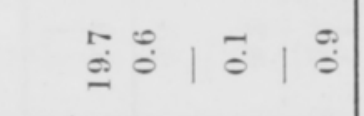 & 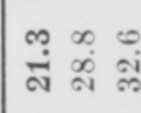 \\
\hline$\bullet$ & 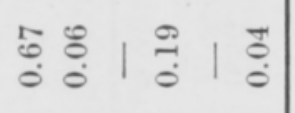 & \&̊ & 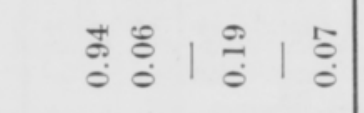 & 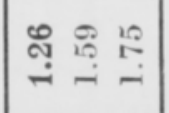 & 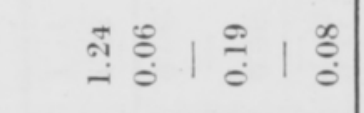 & 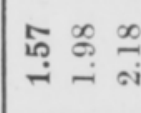 \\
\hline 20 & 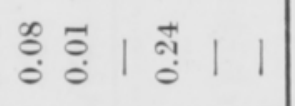 & mְ & 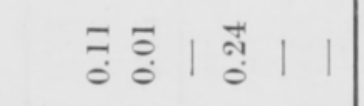 & 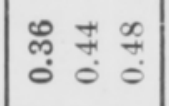 & 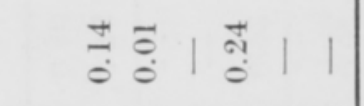 & 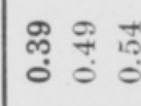 \\
\hline+4 & 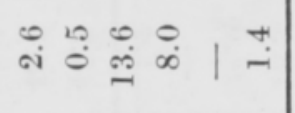 & 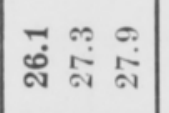 & 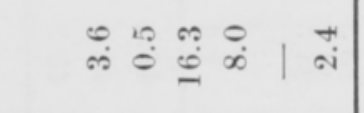 & 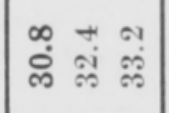 & 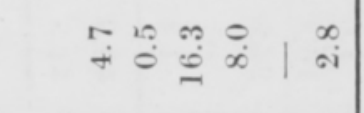 & 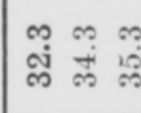 \\
\hline$\infty$ & ભู & 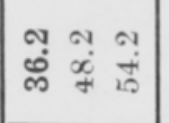 & 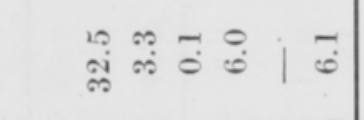 & $\left|\begin{array}{lll}0 & 0 & 0 \\
\dot{\alpha} & \dot{0} & i \\
\end{array}\right|$ & 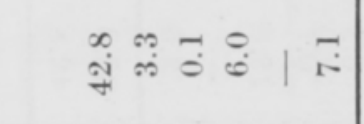 & 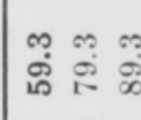 \\
\hline a & 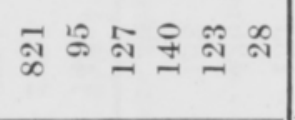 & 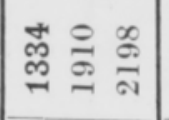 & 동 & 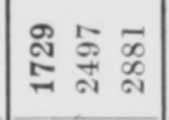 & 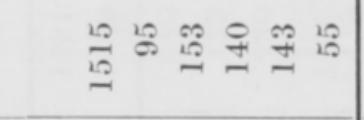 & 그요 \\
\hline- & 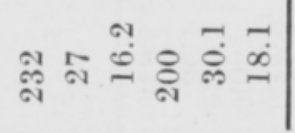 & 111 & 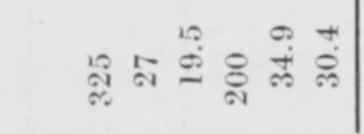 & 11 & 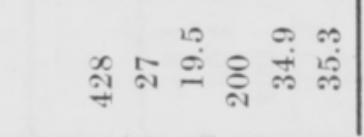 & $1 \mid 1$ \\
\hline 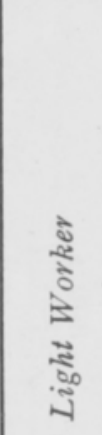 & 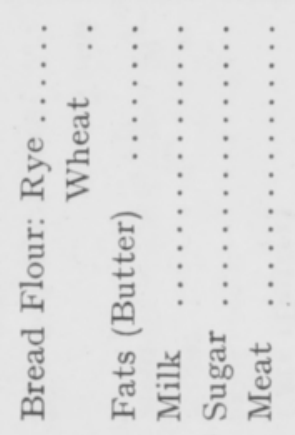 & 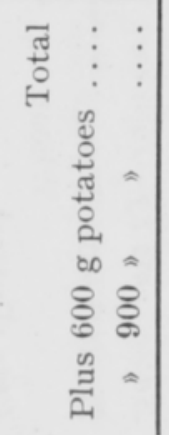 & 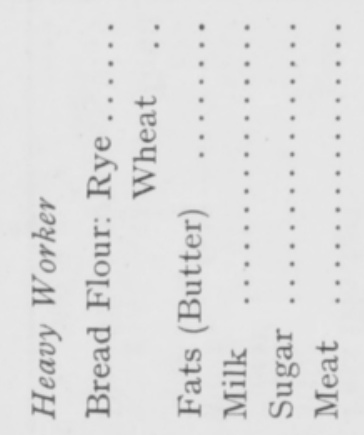 & 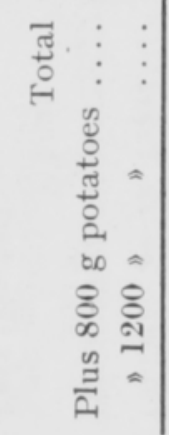 & 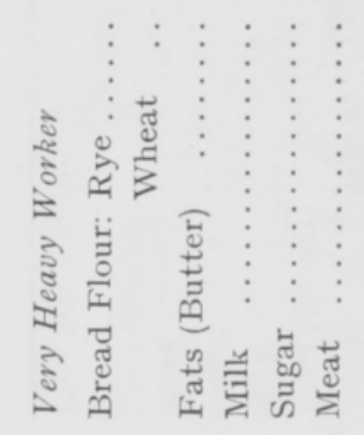 & 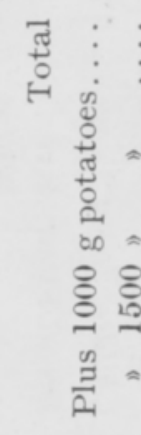 \\
\hline
\end{tabular}




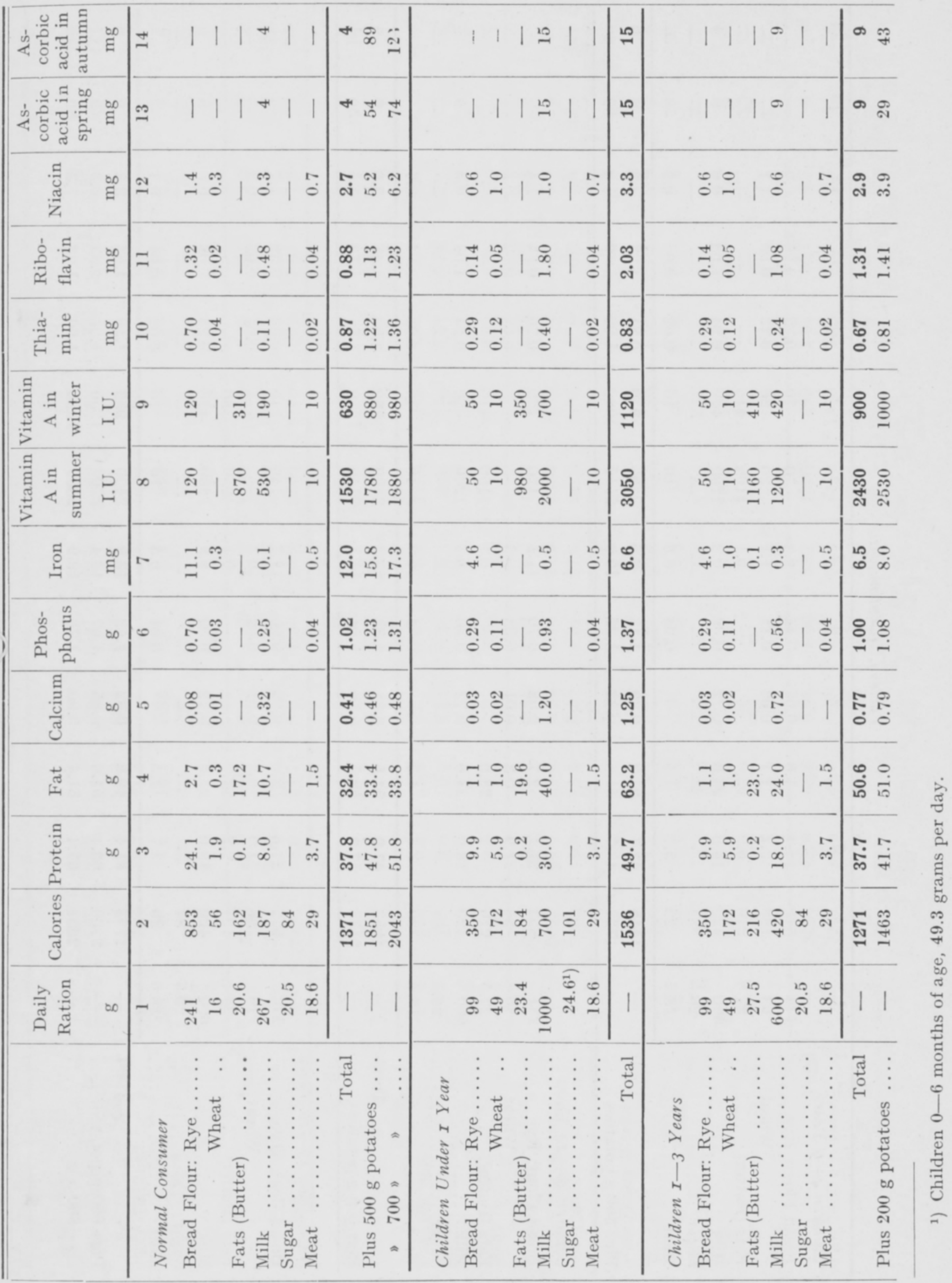




\begin{tabular}{|c|c|c|c|c|c|c|}
\hline ت゙ & $\begin{array}{llllll}1 & 1 & 1 & 0 & 1 & 1\end{array}$ & $\infty 8$ & $\mid \begin{array}{lllll}\mid & \mid & \infty & \mid & 1\end{array}$ & $\sigma$ ప & $|1| \infty \mid 1$ & $\infty \equiv \pm$ \\
\hline 9 & $|1| \infty \mid 1$ & a & $|1| \infty|l|$ & or 8 & $\begin{array}{llllll}1 & 1 & 1 & 0 & 1 & 1\end{array}$ & $\infty \stackrel{\theta}{\infty} \infty$ \\
\hline$\cong$ & 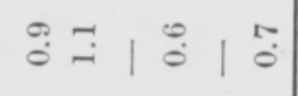 & $\begin{array}{ll}\infty & \infty \\
\infty & +\end{array}$ & $\stackrel{\leftrightarrow}{-} \ddot{0}|\stackrel{0}{0}| \tilde{\theta}$ & $\begin{array}{ll}0 & 10 \\
\text { if } & 10\end{array}$ & $\because \stackrel{\oplus}{0}|\stackrel{\varphi}{0}| \tilde{\theta}$ & 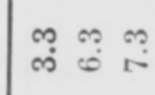 \\
\hline$\Xi$ & 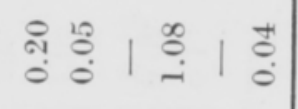 & 䗆 & 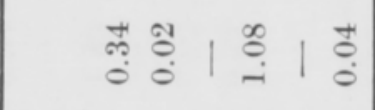 & 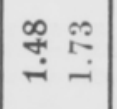 & 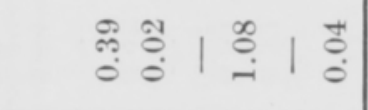 & 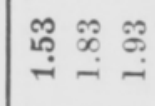 \\
\hline$\varrho$ & 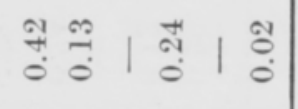 & 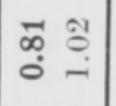 & 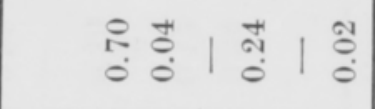 & ○ִ & 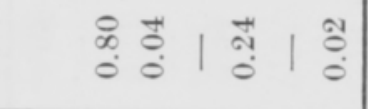 & 윽 을 \\
\hline os & 요욕 & ○ి $\stackrel{0}{\circ} \stackrel{\infty}{\varrho}$ & 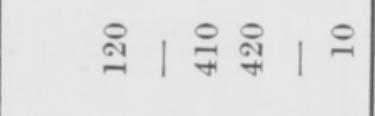 & \& & 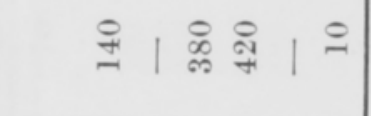 & 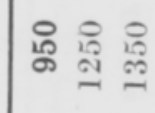 \\
\hline$\infty$ & 요요요 & 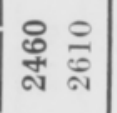 & 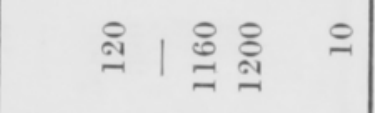 & 号 & 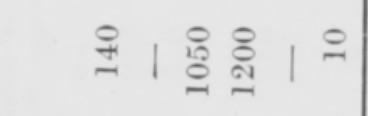 & 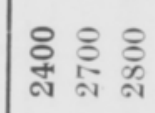 \\
\hline$r$ & $\stackrel{0}{\dot{0}}=\vec{i} \dot{0} \stackrel{\leftrightarrow}{0} \mid \stackrel{10}{\circ}$ & $\stackrel{0}{\infty} \stackrel{0}{\oplus}$ & 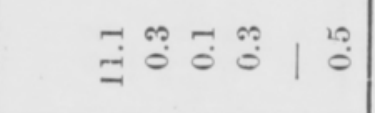 & 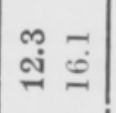 & 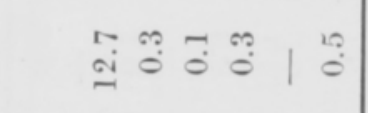 & 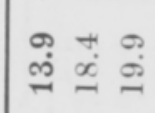 \\
\hline$\bullet$ & 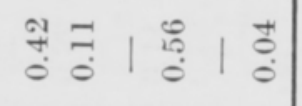 & $\stackrel{m}{\longrightarrow} \stackrel{2}{\rightarrow}$ & 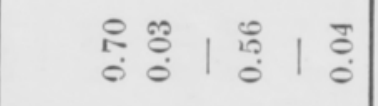 & ஜొ & 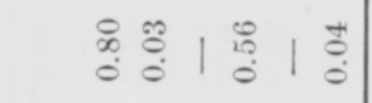 & 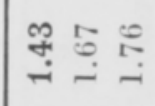 \\
\hline 10 & 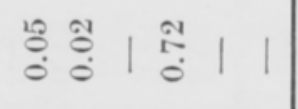 & 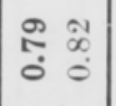 & 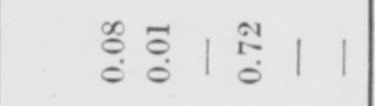 & $\mid \begin{array}{ll}\overrightarrow{0} & 0 \\
\infty & \infty \\
0 & 0\end{array}$ & 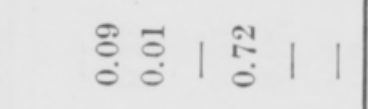 & 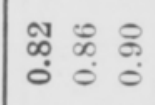 \\
\hline$H$ & 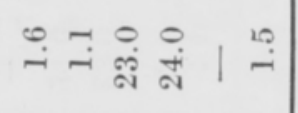 & ஸ़่ & 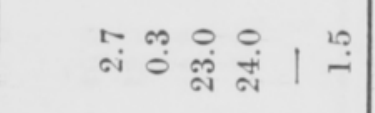 & ำ & 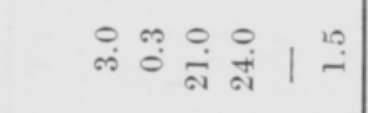 & 我 \\
\hline$\infty$ & 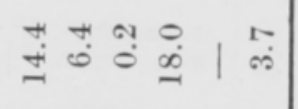 & 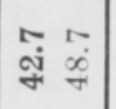 & 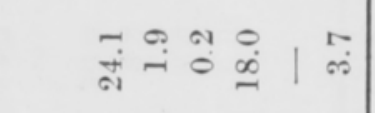 & 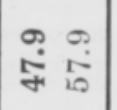 & 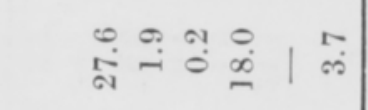 & 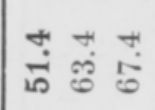 \\
\hline o & 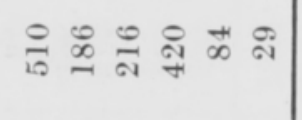 & 字 & 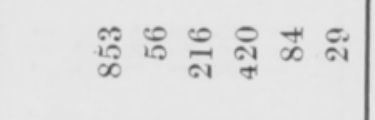 & 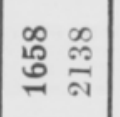 & 点 & 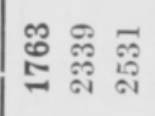 \\
\hline & 节 & 11 & 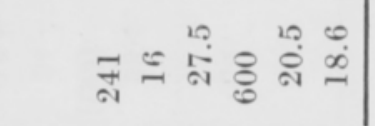 & $1 \mid$ & 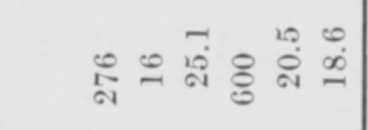 & $1 \mid 1$ \\
\hline 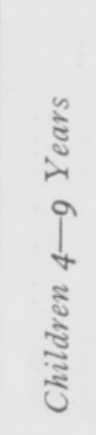 & 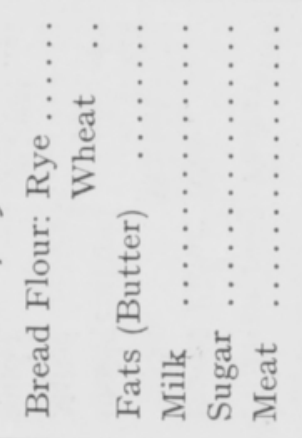 & 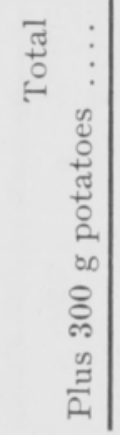 & 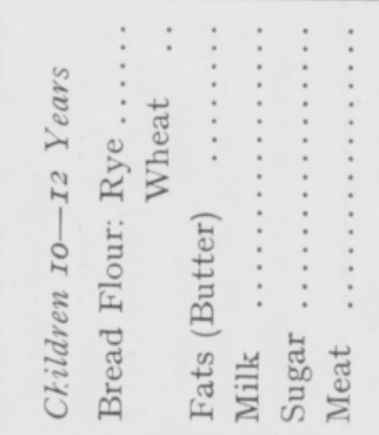 & 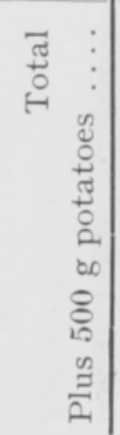 & 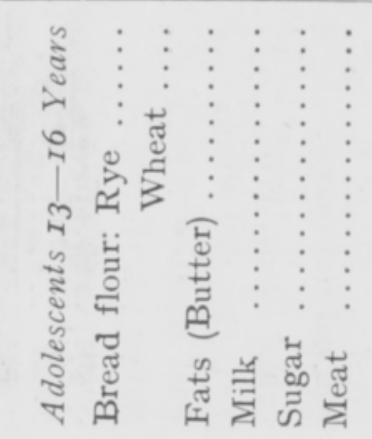 & 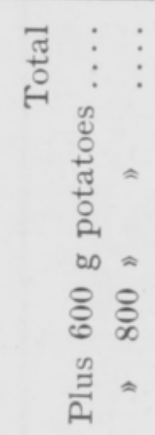 \\
\hline
\end{tabular}




\begin{tabular}{|c|c|c|c|c|c|c|c|}
\hline & $1|1+1|$ & 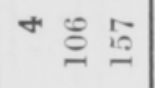 & & $1 \mid 1+1$ & + 욤 & t & $+\frac{5}{5}$ \\
\hline & $11|+1|$ & + పే ప & & $1|1+1|$ & $+\vec{\infty}$ & | $1+$ & + \\
\hline & $=\stackrel{\leftrightarrow}{\sim}|\stackrel{\leftrightarrow}{0}| \stackrel{r}{0}$ & $\begin{array}{lll}0 & 0 & 12 \\
\text { in } & \dot{0} & \stackrel{1}{1}\end{array}$ & & 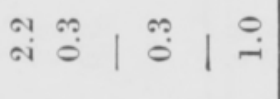 & $\begin{array}{lll}\infty & \infty & \infty \\
\dot{\infty} & \stackrel{\infty}{r} & \infty\end{array}$ & $\stackrel{r}{\sim} \stackrel{\leftrightarrow}{0}|\stackrel{\leftrightarrow}{0}| \stackrel{\oplus}{-}$ & 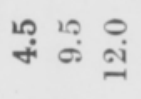 \\
\hline & 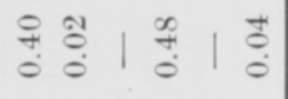 & 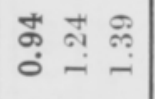 & & 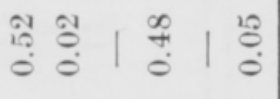 & 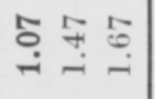 & 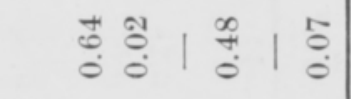 & 꼬 동 \\
\hline & 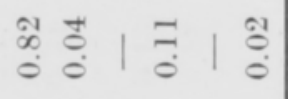 & 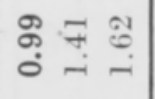 & & 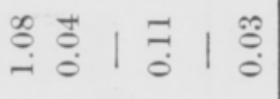 & 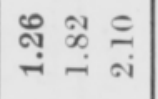 & ஜै & ํㅗ \\
\hline & 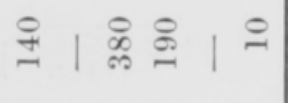 & 옹요ㅇㅛㅡㄹ & & 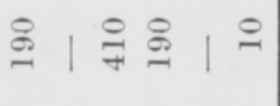 & ○े & คิ & 品员 怘 \\
\hline & 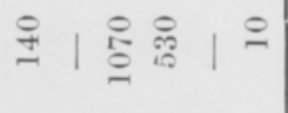 & 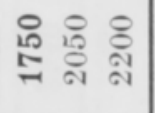 & & 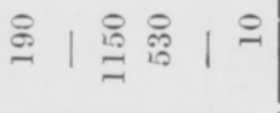 & $\begin{array}{lll}0 & 0 & 0 \\
\infty & \infty & \infty \\
\infty & \mathbb{1} & + \\
-1 & \text { N } & \text {. }\end{array}$ & 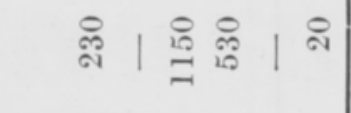 & 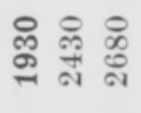 \\
\hline & 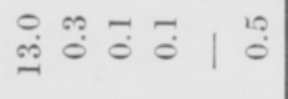 & 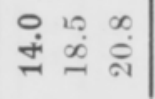 & & 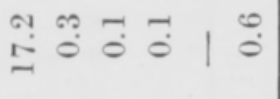 & 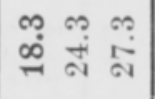 & $\stackrel{\leftrightarrow}{\leftrightarrow}_{\stackrel{\infty}{0}}^{\infty} \stackrel{0}{0} \overrightarrow{0} \overrightarrow{0} \mid \stackrel{\infty}{0}$ & 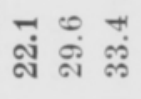 \\
\hline & 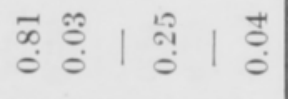 & 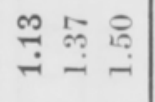 & & 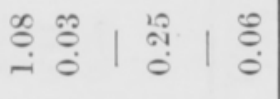 & 采望它 & 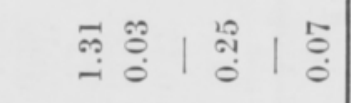 & 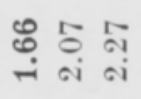 \\
\hline & 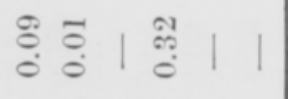 & 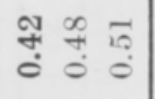 & & 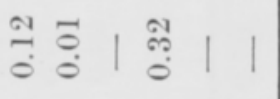 & 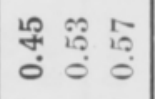 & $\stackrel{120}{0} \stackrel{0}{0}|\stackrel{\oplus}{0}|$ & $\begin{array}{lll}\infty & \infty & 0 \\
+ & 0 & 0 \\
0 & 0 & 0 \\
0 & 0 & 0\end{array}$ \\
\hline & 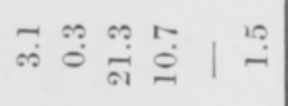 & 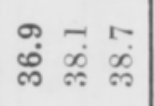 & & 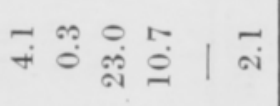 & 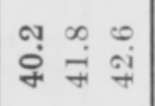 & 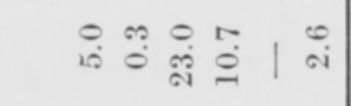 & 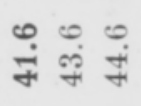 \\
\hline & 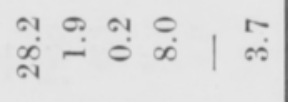 & $\begin{array}{lll}0 & 0 & 0 \\
\text { มี } & \stackrel{+}{10} & \dot{8}\end{array}$ & & 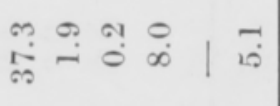 & $\begin{array}{lll}0 & 10 & 19 \\
\text { iิ } & 0 & 0 \\
0 & 0 & 0\end{array}$ & 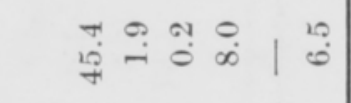 & 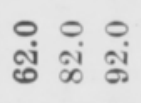 \\
\hline & 号 & 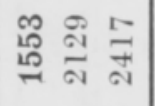 & & 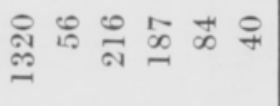 & 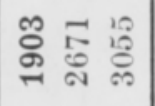 & 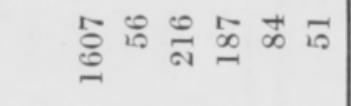 & 灾 \\
\hline & 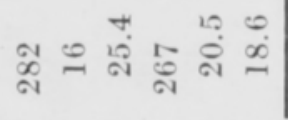 & 111 & & ๓⿴囗十) & 111 & 苟 & 11 \\
\hline & 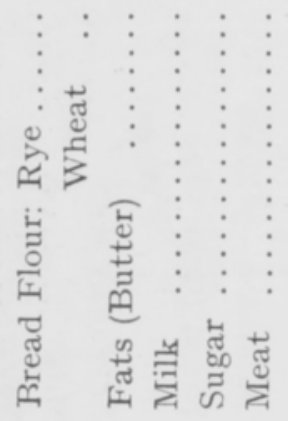 & 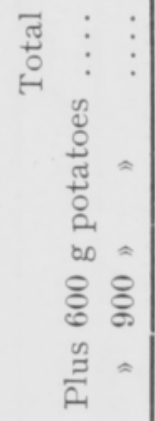 & 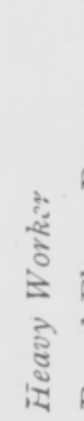 & 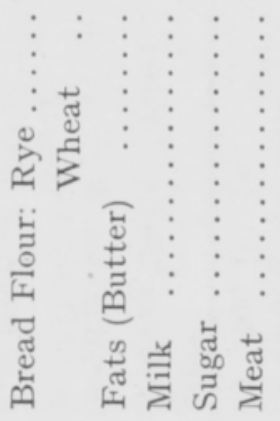 & 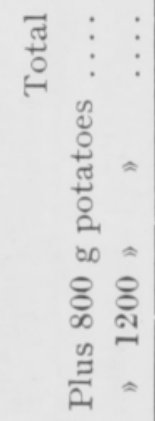 & 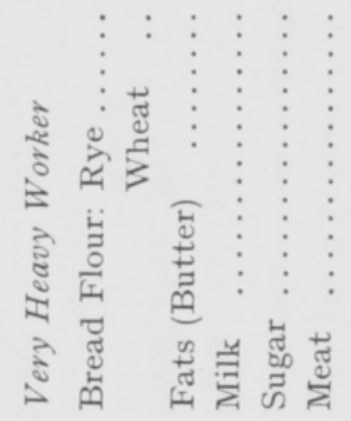 & 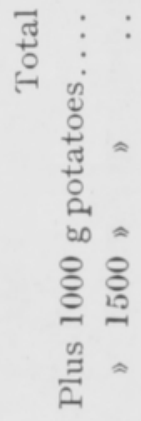 \\
\hline
\end{tabular}




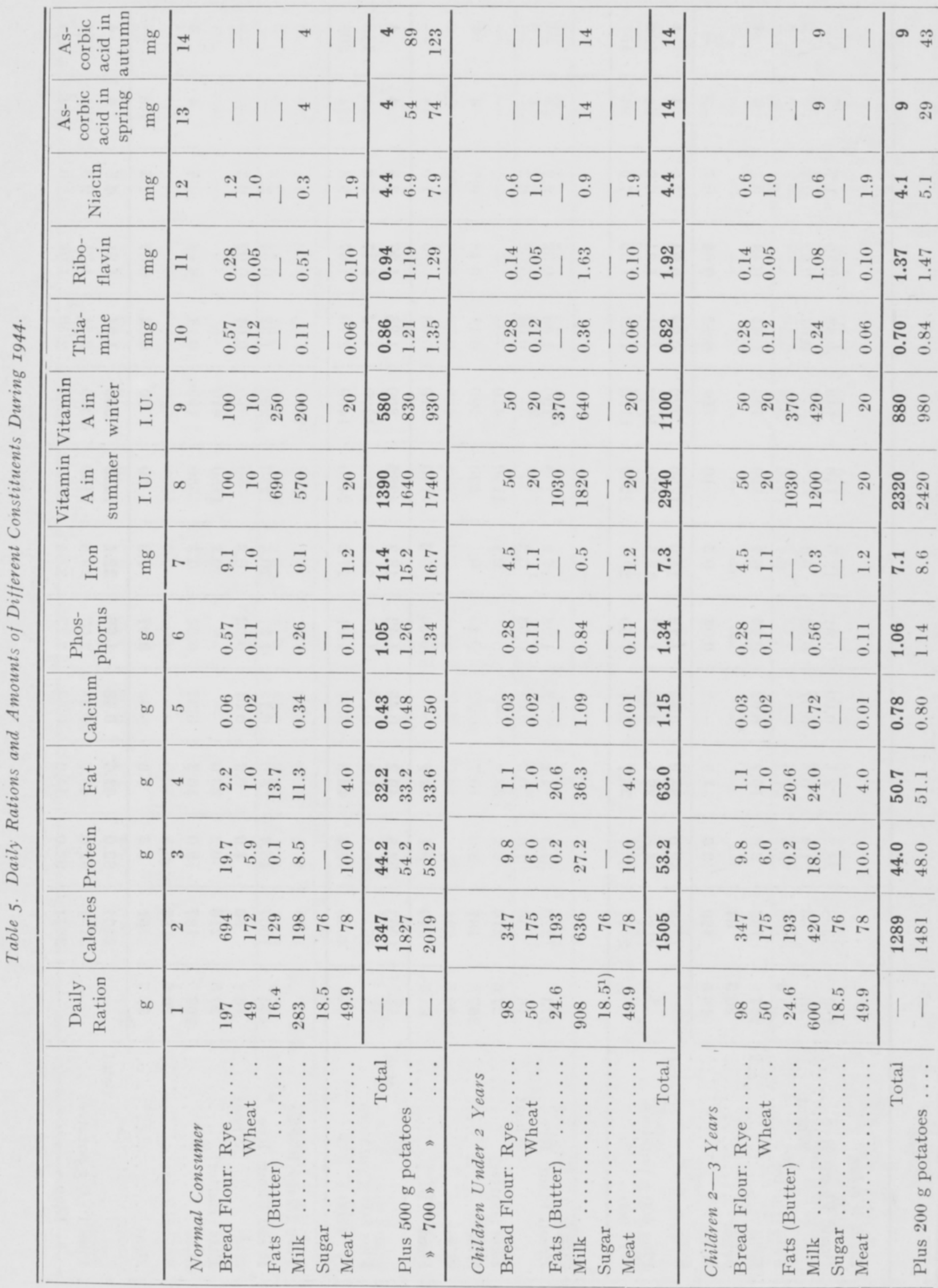




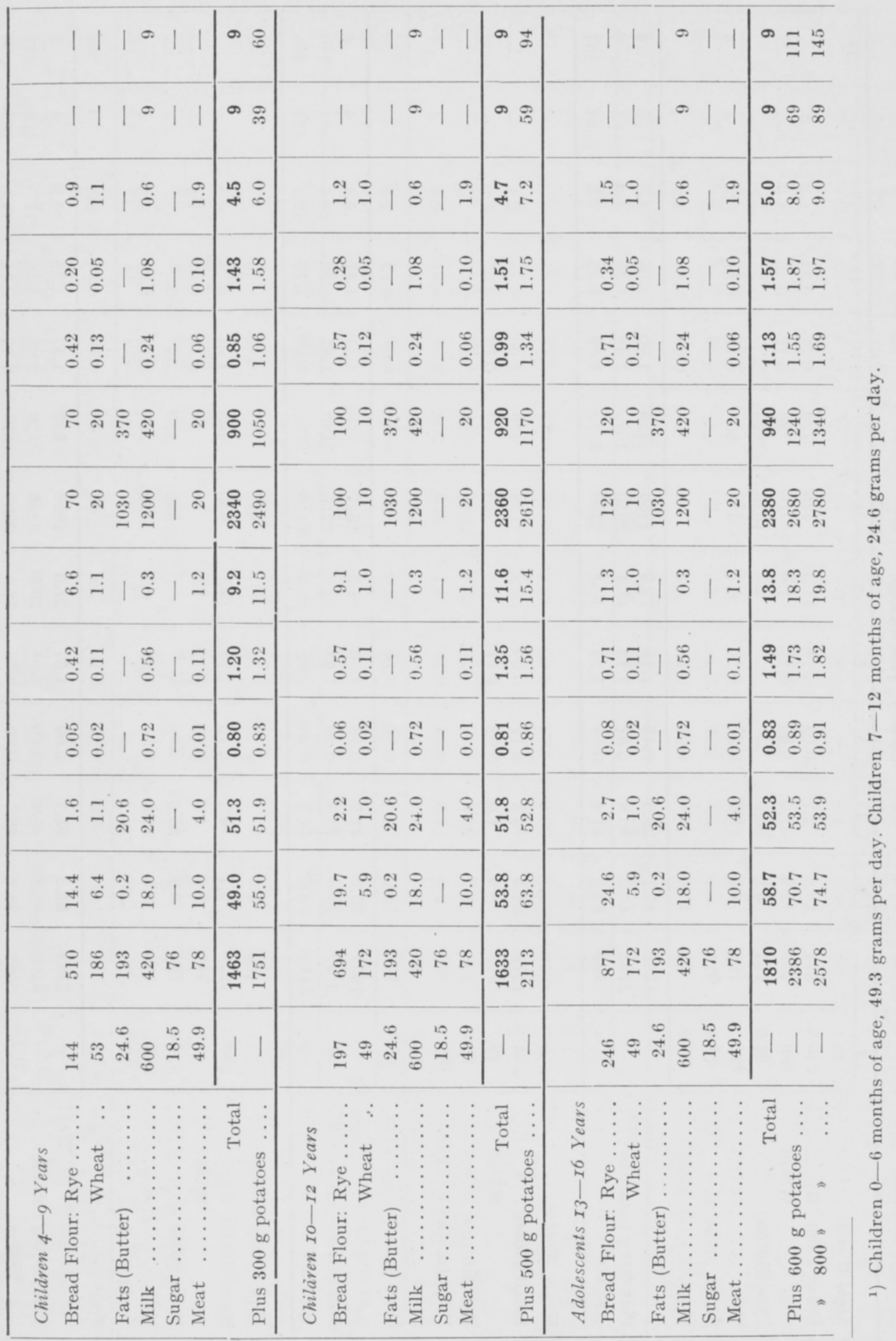




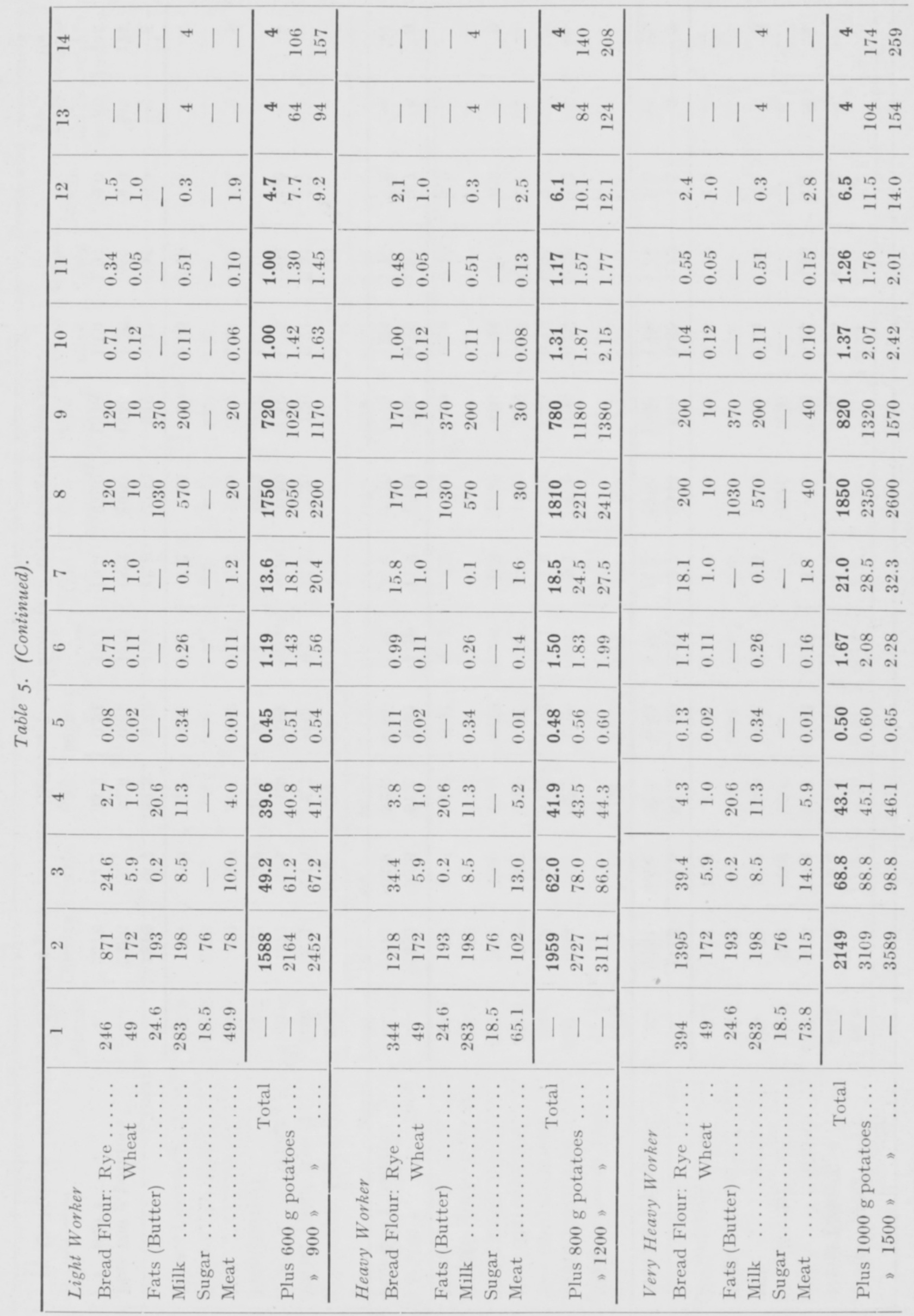




\begin{tabular}{|c|c|c|c|c|c|c|c|c|c|}
\hline 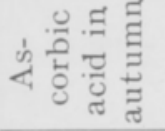 & $\stackrel{\infty}{\sharp}$ & \pm & ||$|m| 1$ & 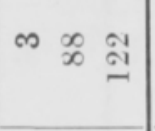 & & | | | & $\Xi$ & $|1| \theta_{1} \mid$ & $\infty \stackrel{9}{9}$ \\
\hline के & $\stackrel{\infty}{a}$ & $\cong$ & ||$|m| 1$ & ๓ & & | | | & \pm & $|1| \infty \mid 1$ & $\sigma$ คి \\
\hline 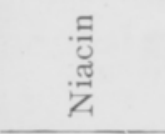 & $\stackrel{\infty}{\sharp}$ & $\cong$ & 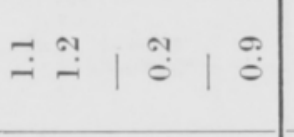 & $\begin{array}{lll}+ & 0 & 9 \\
\text { is } & \text { is } & 0\end{array}$ & & $\stackrel{\theta}{0}-1 \stackrel{\theta}{0} \mid \stackrel{\theta}{0}$ & $\dot{\oplus}$ & $\stackrel{0}{0} \stackrel{0}{-}|\stackrel{\varphi}{0}| \stackrel{0}{0}$ & $\vec{\infty} \overrightarrow{+}$ \\
\hline$\frac{1}{8} \stackrel{\equiv}{=}$ & $\stackrel{\infty}{\Xi}$ & $=$ & 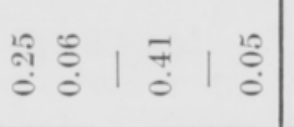 & ำ & & 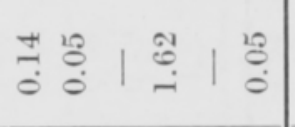 & $\stackrel{\infty}{\infty}$ & 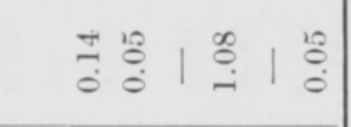 & ભิ ঙำ \\
\hline 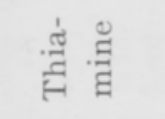 & $\stackrel{\infty}{\Xi}$ & $\cong$ & 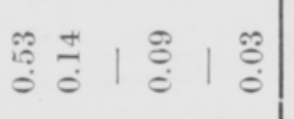 & 足 & & 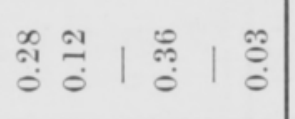 & ז & 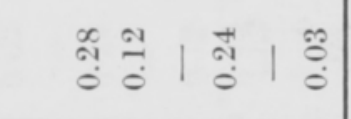 & 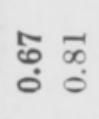 \\
\hline 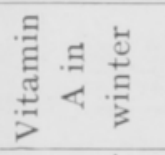 & : & 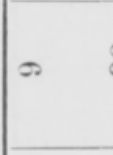 & 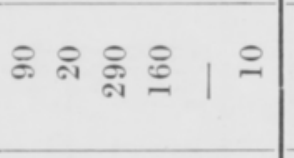 & 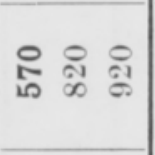 & & 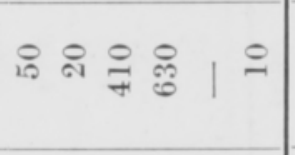 & 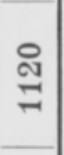 & 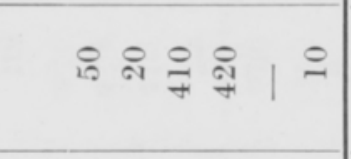 & ๘융 \\
\hline $\begin{array}{lll}\stackrel{\Xi}{\Xi} \\
\underset{J}{J} \\
\vdots\end{array}$ & $\dot{\rho}$ & $\infty$ & 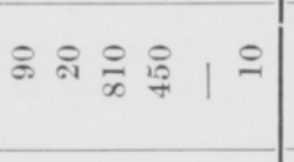 & 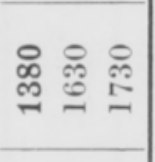 & & 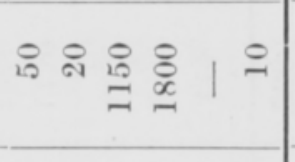 & ळ్లి & 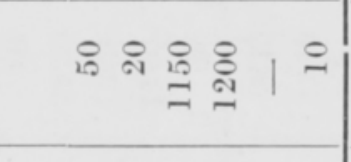 & 前 \\
\hline$\stackrel{5}{\Xi}$ & $\stackrel{\infty}{\Xi}$ & ro & $\vec{\infty} \stackrel{\leftrightarrow}{-}|\overrightarrow{0}| \stackrel{0}{0}$ & 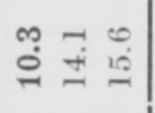 & & 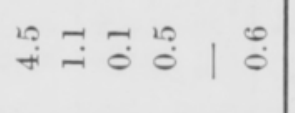 & $\begin{array}{l}\infty \\
\dot{\omega}\end{array}$ & 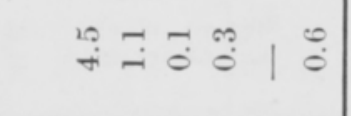 & $\stackrel{\oplus \rightarrow}{\infty} \vec{\infty}$ \\
\hline 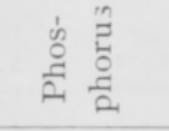 & to & 0 & 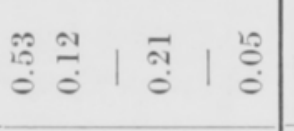 & 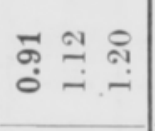 & & 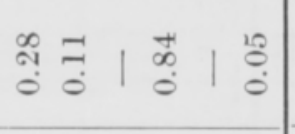 & 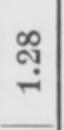 & 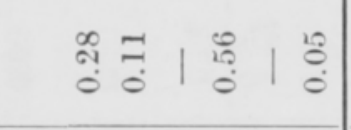 & 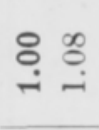 \\
\hline हี & 60 & 10 & 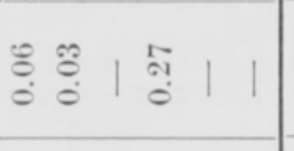 & 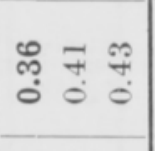 & & 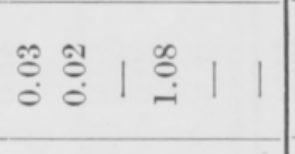 & $\stackrel{m}{\rightarrow}$ & 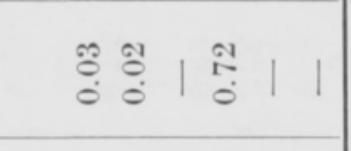 & 둥 \\
\hline 氠 & $\infty$ & + & 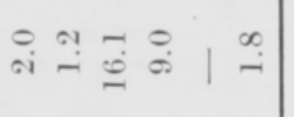 & 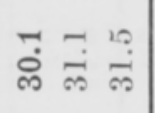 & & 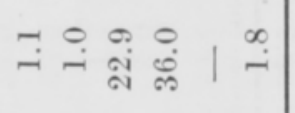 & $\begin{array}{l}\infty \\
\text { తิ่ } \\
\text { in }\end{array}$ & 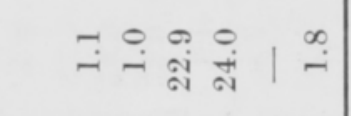 & 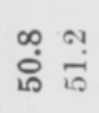 \\
\hline $\begin{array}{l}\frac{1}{0} \\
0 \\
0 \\
0\end{array}$ & $\infty$ & $\infty$ & $\begin{array}{llllll}\because & 0 & - & \infty & 0 \\
\infty & 0 & 0 & 0 & +\end{array}$ & 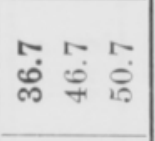 & & 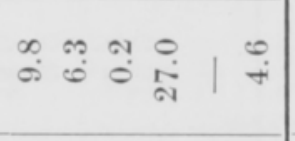 & 安 & $\begin{array}{llllll}\infty & \oplus & \oplus & 0 & \varphi \\
\infty & 0 & 0 & \infty & \mid & + \\
0 & & & \end{array}$ & 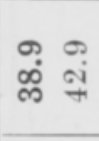 \\
\hline$\frac{\infty}{3}$ & & $a$ & ప艹 & 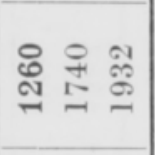 & & 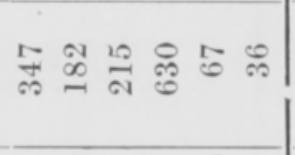 & 壱 & 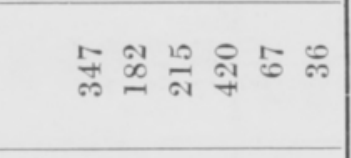 & 농 \\
\hline तี & $\infty$ & - & 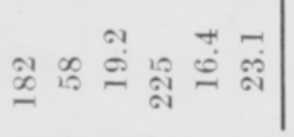 & $1 \mid 1$ & & 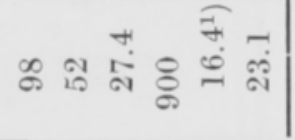 & 1 & 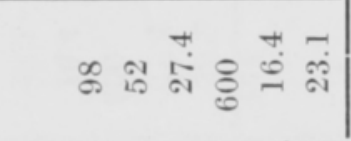 & 11 \\
\hline & & 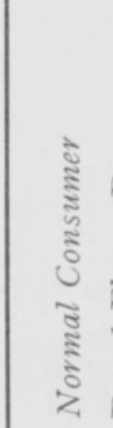 & 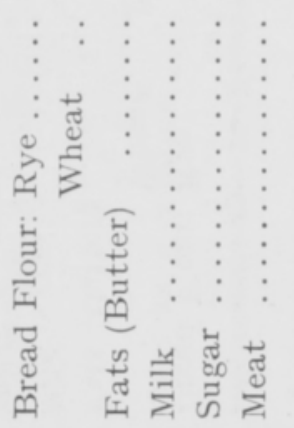 & 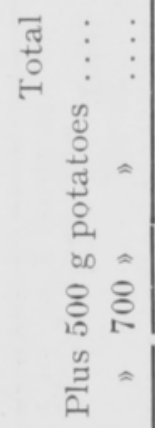 & 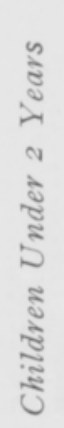 & 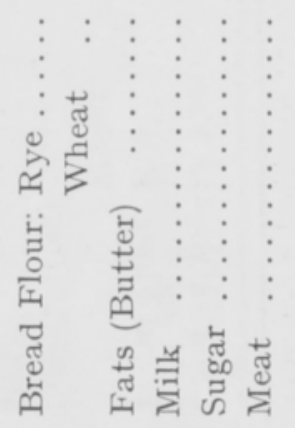 & $\begin{array}{l}\text { సే } \\
\stackrel{0}{0} \\
+\end{array}$ & 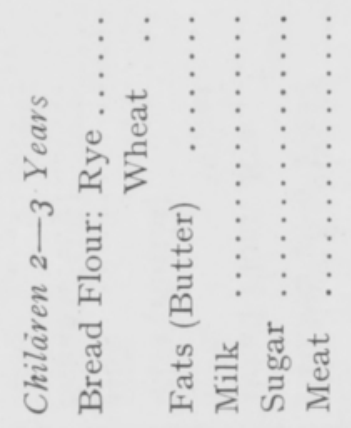 & 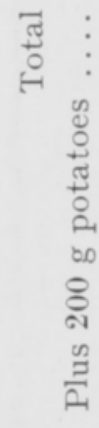 \\
\hline
\end{tabular}




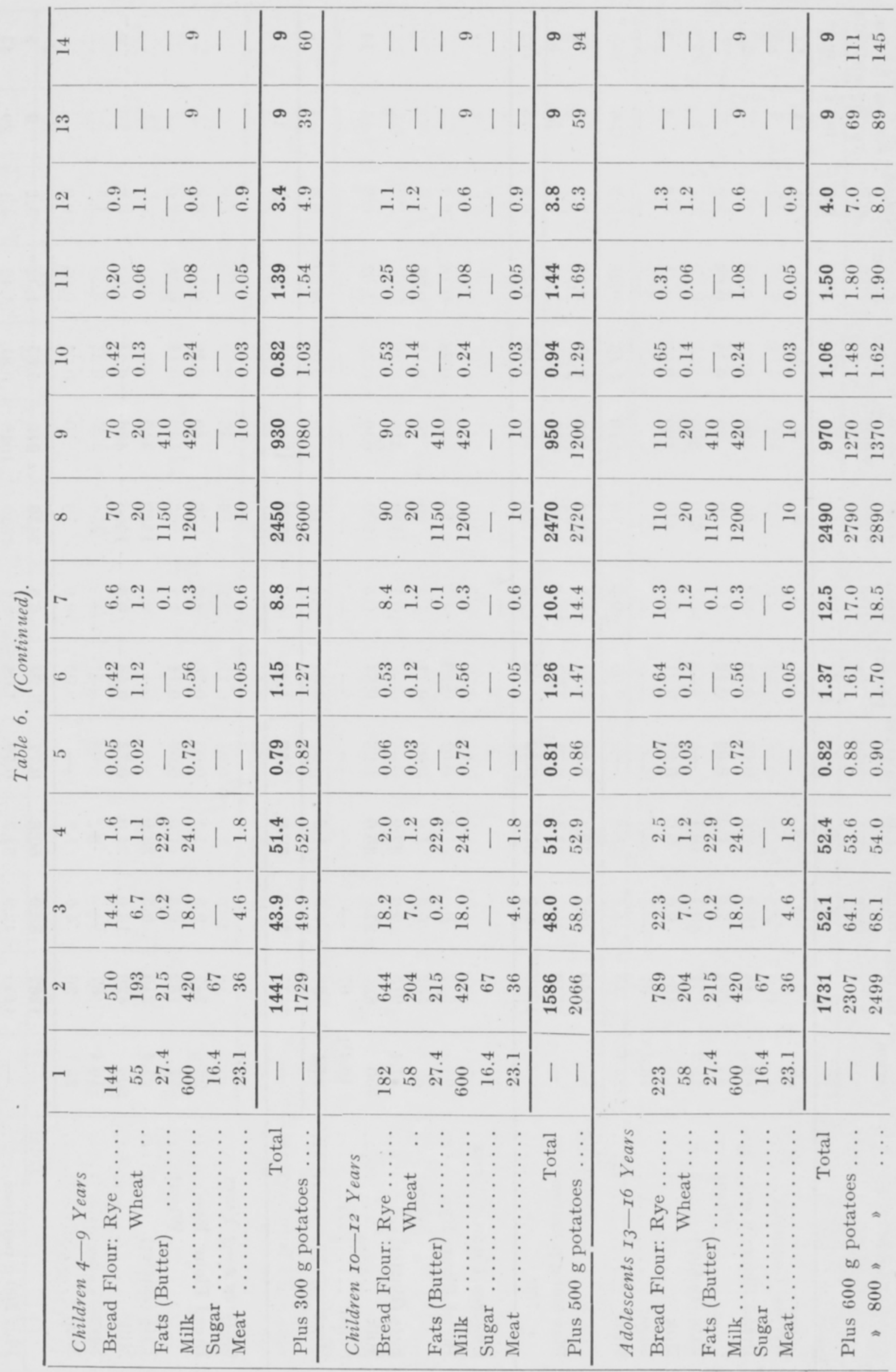




\begin{tabular}{|c|c|c|c|c|c|}
\hline||$|\infty| 1$ & 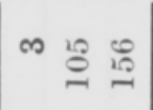 & $|1| \infty \mid 1$ & m & ||$|m| 1 \mid$ & 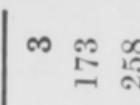 \\
\hline$|1| \infty$ & $\infty \cong$ & $|1| \infty|1|$ & $\infty \approx$ & $|1| \infty|1|$ & $\infty \stackrel{0}{g}$ \\
\hline 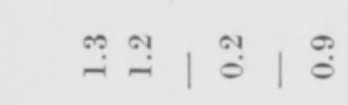 & \begin{tabular}{ccc|}
0 & 0 & -7 \\
$\dot{\infty}$ & $\dot{\infty}$ & $\infty$
\end{tabular} & 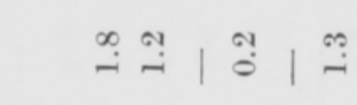 & $\begin{array}{lll}10 & 10 & 10 \\
+\infty & \infty & 0\end{array}$ & 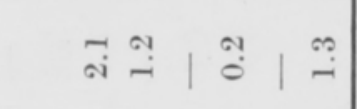 & 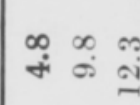 \\
\hline تூ & 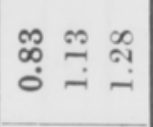 & 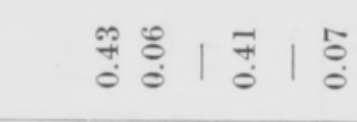 & 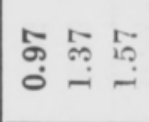 & 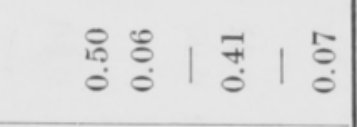 & 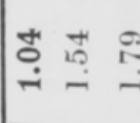 \\
\hline 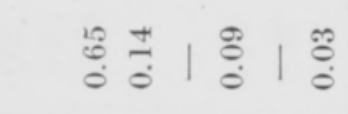 & क् & 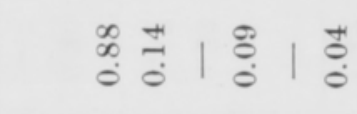 & 量 & 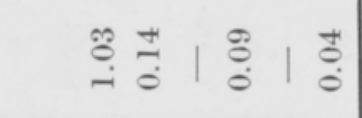 & \%ุำ \\
\hline 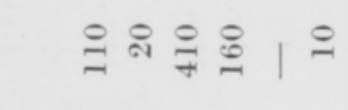 & 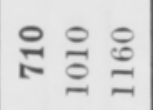 & 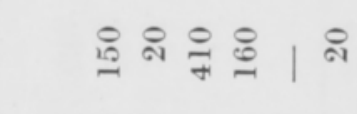 & 俤总 & 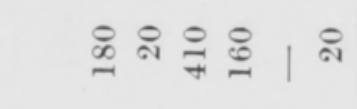 & 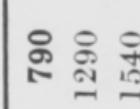 \\
\hline 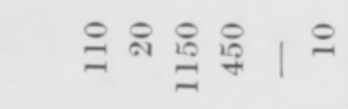 & 呈早点 & 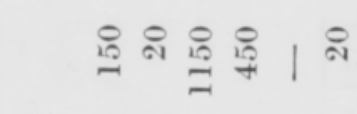 & 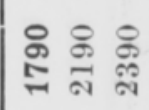 & 赵怘员品 & 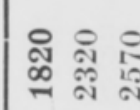 \\
\hline 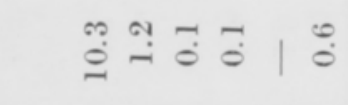 & 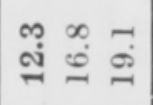 & 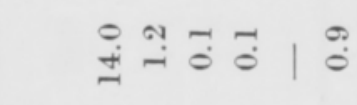 & 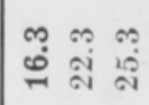 & $\stackrel{\leftrightarrow}{\leftrightarrow} \stackrel{\oplus}{-} \overrightarrow{0} \overrightarrow{0} \mid \stackrel{\oplus}{0}$ & 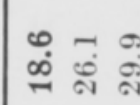 \\
\hline 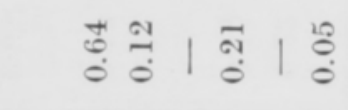 & 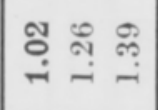 & 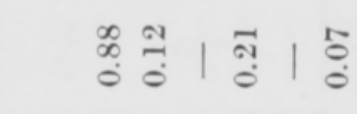 & 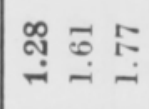 & 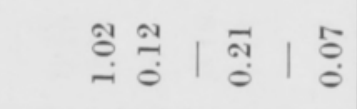 & 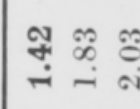 \\
\hline $\begin{array}{l}\text { Do } \\
0 \\
0\end{array}$ & 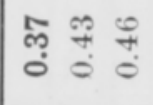 & 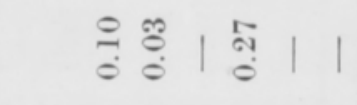 & 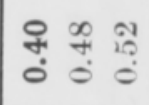 & 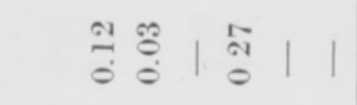 & 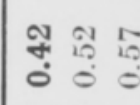 \\
\hline 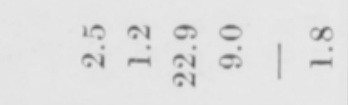 & 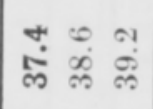 & 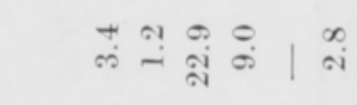 & 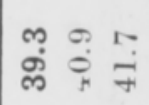 & 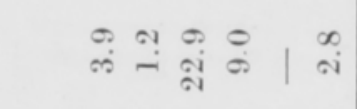 & 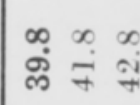 \\
\hline 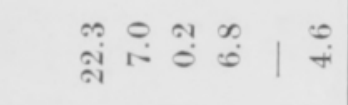 & 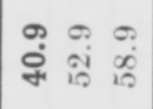 & 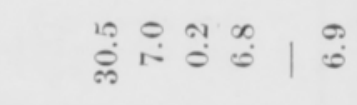 & 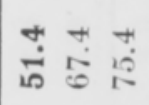 & 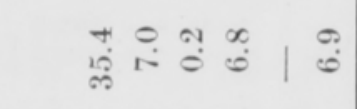 & 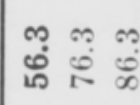 \\
\hline 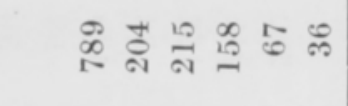 & 里电 & 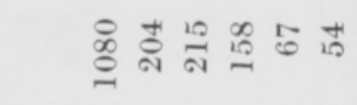 & 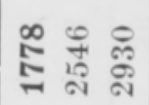 & 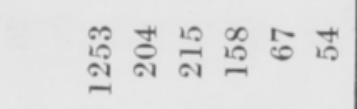 & $\overrightarrow{\mathrm{g}} \overline{\mathrm{s}}$ \\
\hline 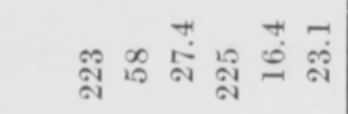 & $\begin{array}{lll}1 & 1 & 1\end{array}$ & 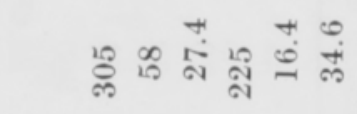 & 111 & 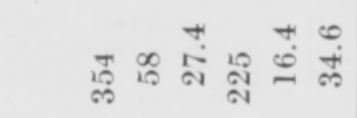 & 111 \\
\hline 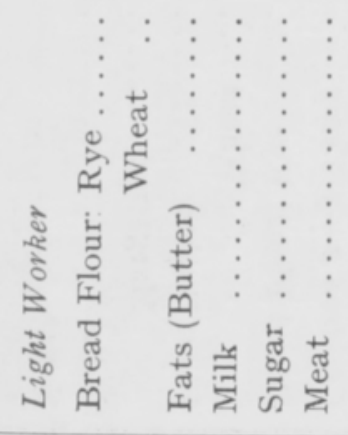 & 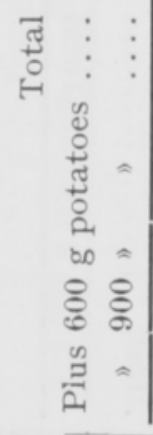 & 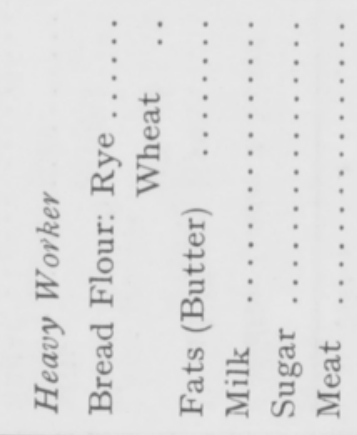 & 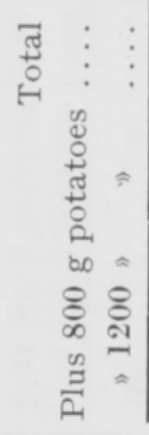 & 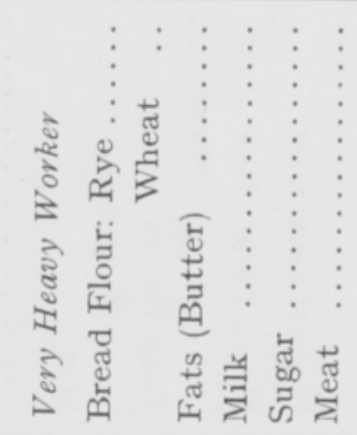 & 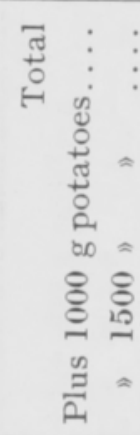 \\
\hline
\end{tabular}


है

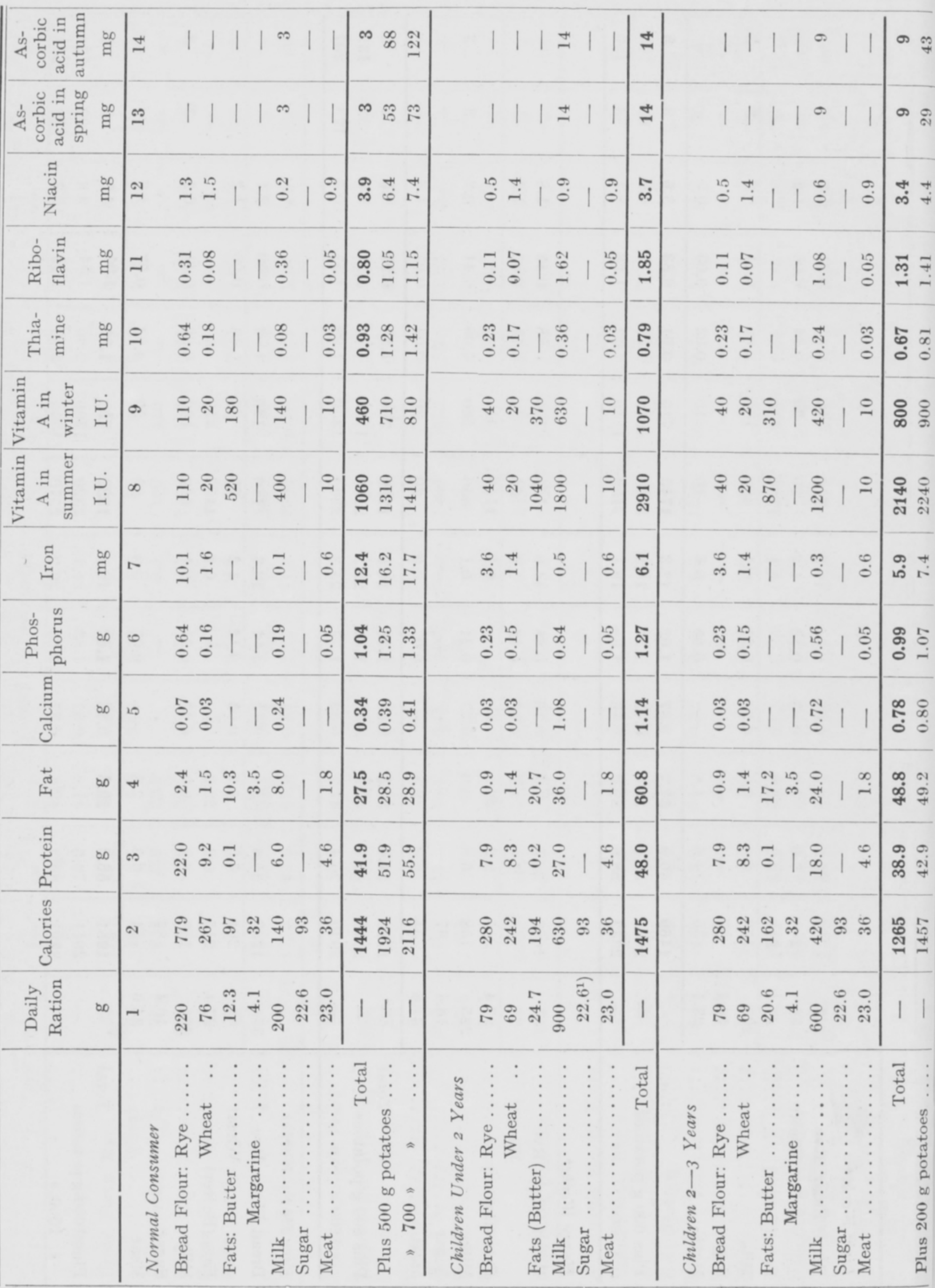




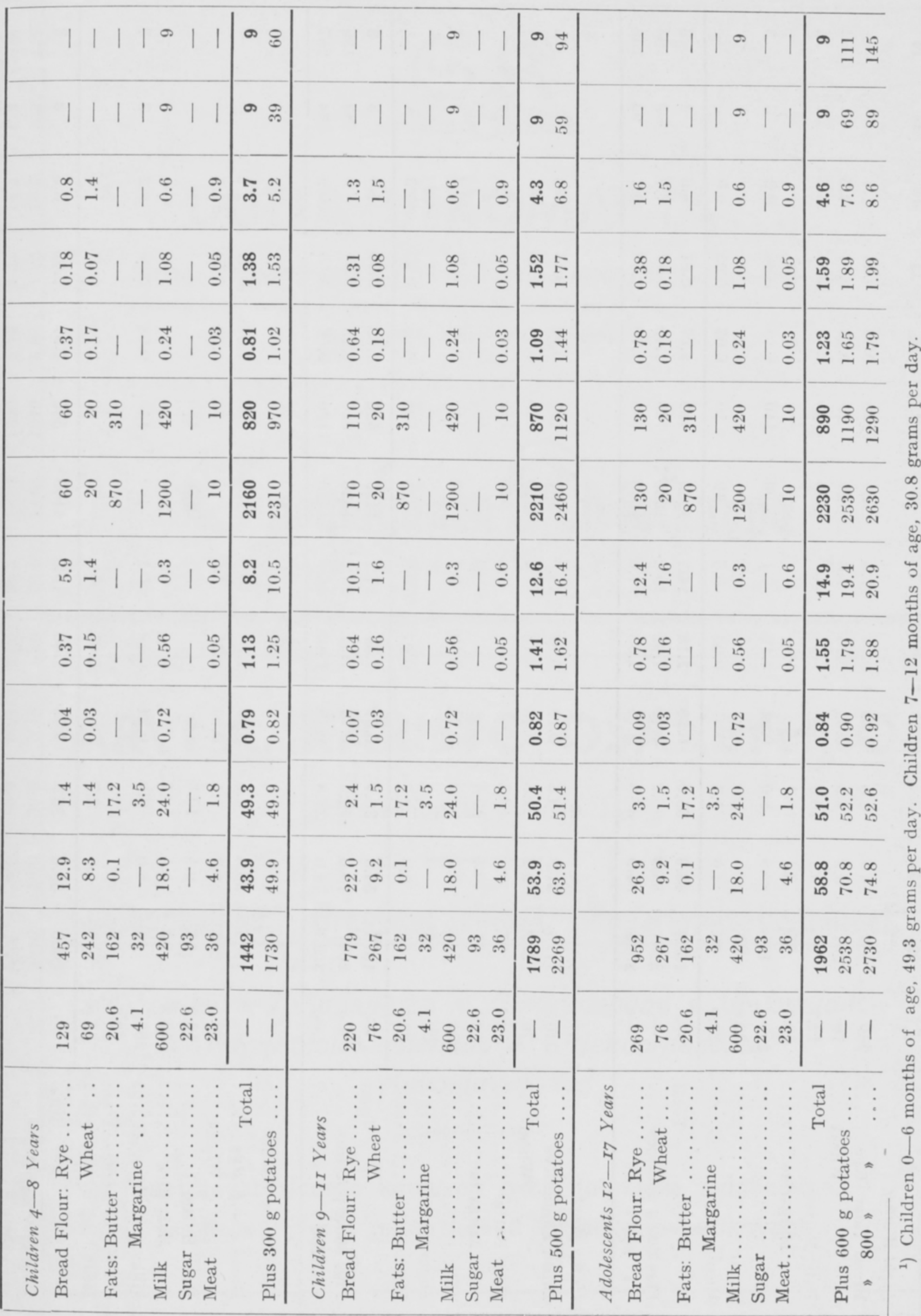




\begin{tabular}{|c|c|c|c|c|c|c|}
\hline & $|1||\infty| 1$ & $\infty \begin{array}{lll}\infty & 20 & 0 \\
& \stackrel{1}{=} & 10\end{array}$ & 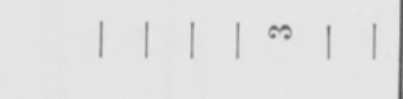 & 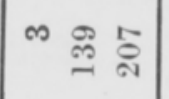 & $1 \mid 1$ & 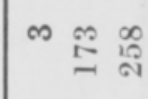 \\
\hline$\dddot{9}$ & ||||$\infty||$ & $\infty \Re$ & $|1||\infty| 1$ & $\infty \stackrel{\infty}{\infty}$ & $1 \mid 1$ & $\infty \stackrel{\dddot{g}}{\mathscr{g}}$ \\
\hline$\stackrel{-19}{2}$ & $\stackrel{\oplus}{-} \stackrel{\leftrightarrow}{-}|| \frac{9}{0} \mid \stackrel{\oplus}{0}$ & 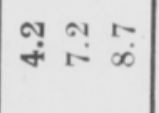 & 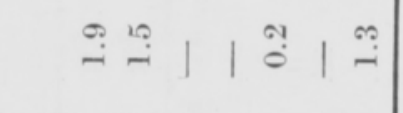 & $\stackrel{\oplus}{+} \underset{\infty}{\infty} \stackrel{\oplus}{\varrho}$ & 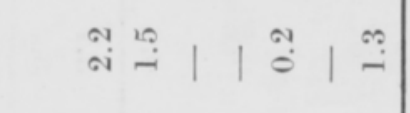 & 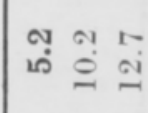 \\
\hline$\approx$ & $\stackrel{\infty}{\stackrel{\infty}{0}} \underset{0}{0} \mid$ & 怘 & 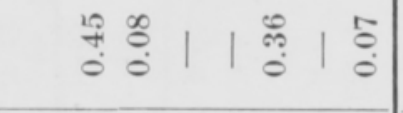 & 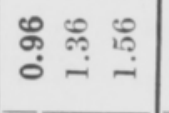 & 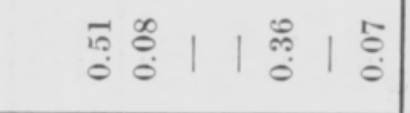 & 옥 옹 \\
\hline$\cong$ & 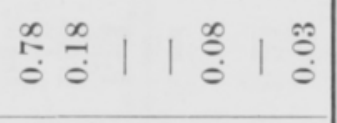 & 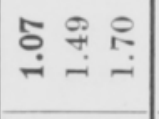 & 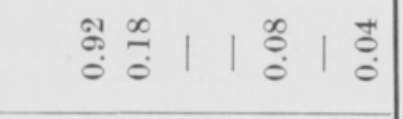 & 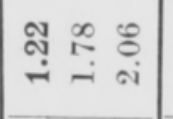 & 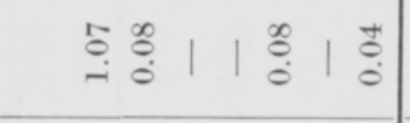 & 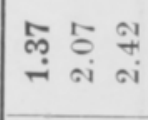 \\
\hline os & 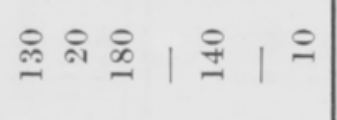 & 总 & 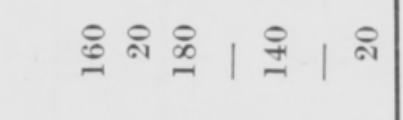 & ㅇํํ 용 ్ㅗㄱ & 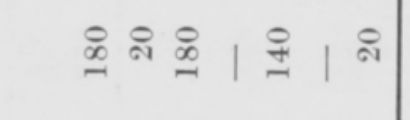 & 웜웡 욤 \\
\hline$\infty$ & 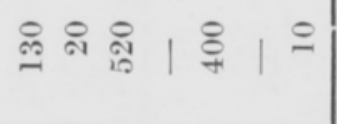 & $\begin{array}{lll}0 & 0 & 0 \\
0 & \infty & 0 \\
0 & 9 & \text { i } \\
-1 & -1\end{array}$ & 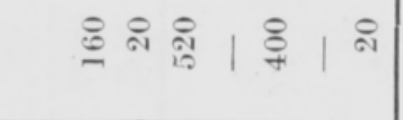 & 오요오ำ & 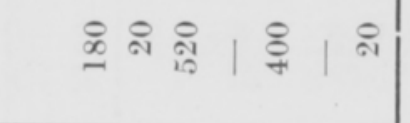 & 웅요 \\
\hline r & $\stackrel{+}{\rightarrow} \stackrel{\varphi}{-}|| \overrightarrow{0} \mid \stackrel{0}{0}$ & 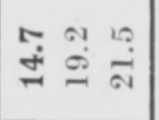 & 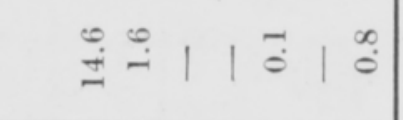 & こ & $\stackrel{\oplus}{\bullet} \stackrel{0}{-}|| \overrightarrow{0}|\stackrel{\infty}{0}|$ & से \\
\hline$\bullet$ & $\stackrel{\infty}{\stackrel{\infty}{0}} \frac{0}{0}|| \frac{9}{0} \mid \stackrel{10}{0}$ & 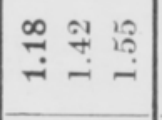 & 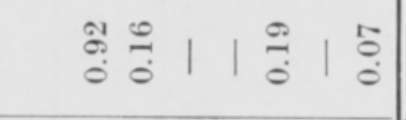 & 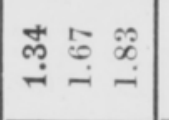 & 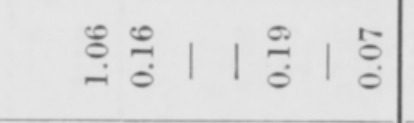 & $\begin{array}{ccc}\infty & 0 & 9 \\
\dot{\sim} & \infty & 0 \\
-i & \text { ii }\end{array}$ \\
\hline 10 & 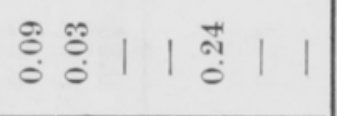 & 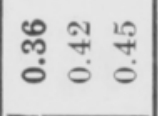 & 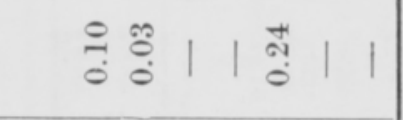 & ஸొ & 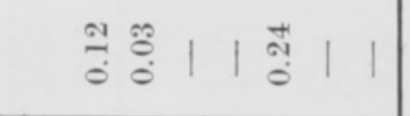 & 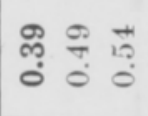 \\
\hline$H$ & 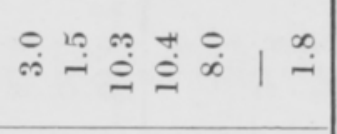 & 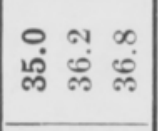 & ஸि & $\begin{array}{lll}+ & 0 & \infty \\
\dot{0} & \infty & \infty \\
\infty & \infty & \infty \\
& \infty & \infty\end{array}$ & 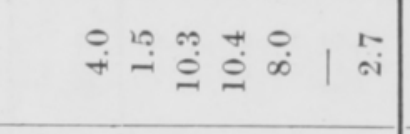 & 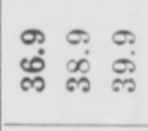 \\
\hline$\infty$ & 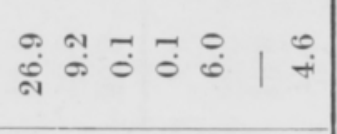 & $\begin{array}{lll}9 & 0 & 0 \\
0 & \infty & 0 \\
\dot{+} & 0 & 0 \\
\end{array}$ & 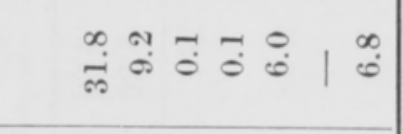 & $\begin{array}{lll}0 & 0 & 0 \\
\dot{1} & 0 & 0 \\
1 & 0 \\
1\end{array}$ & 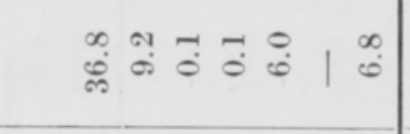 & 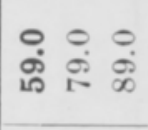 \\
\hline$\infty$ & 今。 & 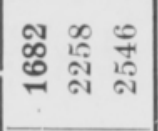 & $\stackrel{\mathscr{N}}{=}$ & 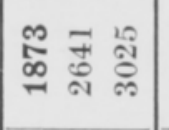 & 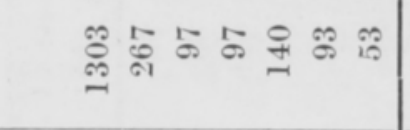 & 잉요용 \\
\hline - & 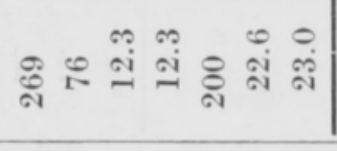 & 111 & 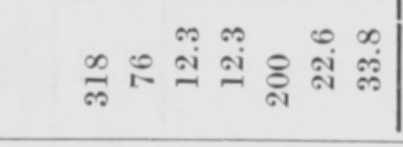 & $1 \mid 1$ & 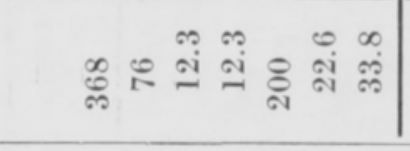 & $1 \mid$ \\
\hline$\underbrace{\frac{1}{4}}$ & 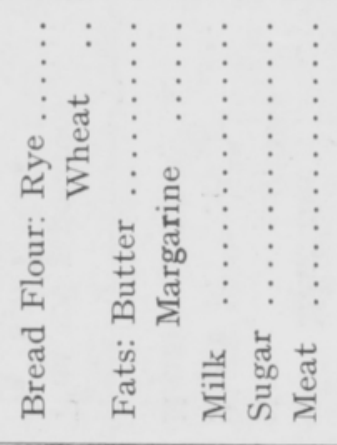 & 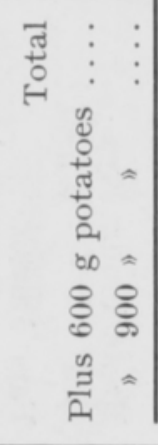 & 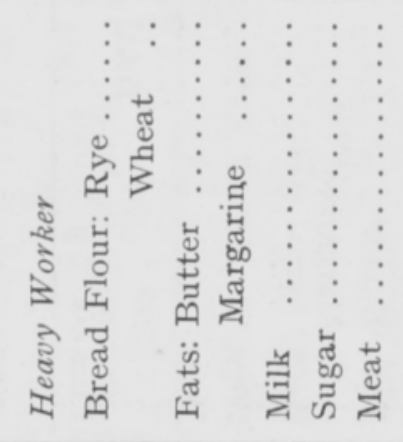 & 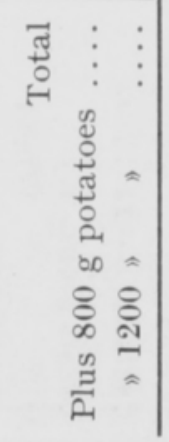 & 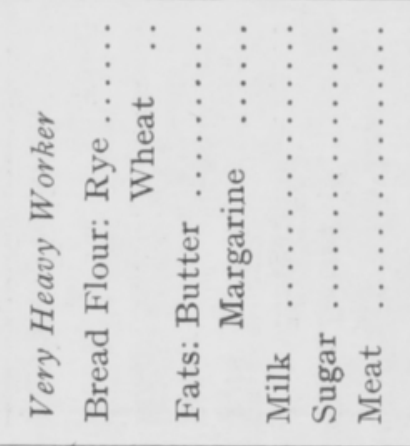 & 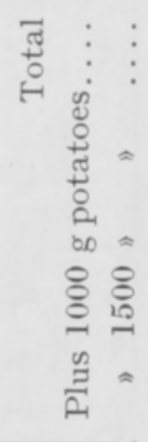 \\
\hline
\end{tabular}

